\title{
Photometric properties of comet 67P/Churyumov-Gerasimenko from VIRTIS-M onboard Rosetta
}

\author{
M. Ciarniello ${ }^{1}$, F. Capaccioni ${ }^{1}$, G. Filacchione ${ }^{1}$, A. Raponi ${ }^{1}$, F. Tosi ${ }^{1}$, M. C. De Sanctis ${ }^{1}$, M. T. Capria ${ }^{1}$, S. Erard ${ }^{2}$, \\ D. Bockelee-Morvan ${ }^{2}$, C. Leyrat ${ }^{2}$, G. Arnold ${ }^{3}$, A. Barucci ${ }^{2}$, P. Beck $^{4}$, G. Bellucci ${ }^{1}$, S. Fornasier ${ }^{2}$, A. Longobardo ${ }^{1}$, \\ S. Mottola ${ }^{3}$, E. Palomba ${ }^{1}$, E. Quirico ${ }^{4}$, and B. Schmitt ${ }^{4}$ \\ 1 IAPS-INAF, via Fosso del Cavaliere, 100, 00133 Rome, Italy \\ e-mail: mauro.ciarniello@iaps.inaf.it \\ ${ }^{2}$ Laboratoire d'Études Spatiales et d'Instrumentation en Astrophysique, Observatoire de Paris/CNRS/Université Pierre et Marie \\ Curie/Université Paris-Diderot, Meudon, 75013 Paris, France \\ 3 Institute of Planetary Research, DLR, 12489 Berlin, Germany \\ ${ }^{4}$ Laboratoire de Planétologie de Grenoble (LPG) - University Joseph Fourier Grenoble, 38041 Grenoble, France
}

Received 13 April 2015 / Accepted 2 July 2015

\begin{abstract}
Aims. We investigate the nucleus photometric properties of the comet 67P/Churyumov-Gerasimenko as observed by the Visible and Infrared Thermal Imaging Spectrometer (VIRTIS) onboard the Rosetta spacecraft. Both full-disk and disk-resolved images of the comet have been analyzed, deriving light and phase curves as well as a photometric reduction of the radiance factor $(I / F)$ to single scattering albedo (SSA) in the $0.4-3.5 \mu \mathrm{m}$ range.

Methods. Hyperspectral cubes from VIRTIS were calibrated and corrected for instrumental artifacts. We computed integrated fluxes from full-disk acquisitions to derive nucleus light curves and phase curves at low phase angles $\left(1.2^{\circ}<\alpha<14.9^{\circ}\right)$. Disk-resolved observations in the phase angle range $27.2^{\circ}<\alpha<111.5^{\circ}$ were reduced to SSA by means of a simplified Hapke model, deriving average spectrophotometric properties of the surface and producing SSA maps at different wavelengths. Spectral phase reddening in the visible (VIS) and infrared (IR) ranges was measured. Finally, full-disk and disk-resolved data were used together to derive a phase curve of the nucleus in the $1.2^{\circ}<\alpha<111.5^{\circ}$ range.

Results. We measure an asymmetric double-peaked light curve that is due to the elongated shape of the nucleus. The average SSA albedo shows a reddish spectrum with a strong absorption feature centered at $3.2 \mu \mathrm{m}$, while the surface exhibits a backscattering behavior. The derived geometric albedo is $A_{\text {geo }}=0.062 \pm 0.002$ at $0.55 \mu \mathrm{m}$, indicating a very dark surface. Phase reddening is significant both in the VIS and IR ranges, and we report spectral slopes of $0.20 / k \AA$ and $0.033 / k \AA$, respectively, after applying photometric reduction. SSA maps indicate that Hapi and Imothep regions are the brightest in the VIS, with the former showing a bluer spectrum with respect to the rest of the surface. The phase curve of the nucleus shows a strong opposition effect, with $\beta=0.077 \pm 0.002$ for $\alpha<15^{\circ}$.
\end{abstract}

Key words. methods: data analysis - techniques: spectroscopic - comets: individual: 67P/Churyumov-Gerasimenko techniques: photometric

\section{Introduction and rationale}

After a cruise phase of more than ten years, the Rosetta spacecraft entered orbit around comet 67P/Churyumov-Gerasimenko (67P) on 6 August 2014. From this date onward, the spacecraft began to escort the comet and will follow it until the end of the nominal mission (December 2015). This allowed very accurate nucleus imaging, making 67P the sixth comet to be directly observed from a spacecraft after 1P/Halley, 9P/Tempel 1, 19P/Borrelly, 81P/Wild 2, and 103P/Hartley 2. The Rosetta orbiter carries eleven instruments, one of which is VIRTIS, the Visible and Infrared Thermal Imaging Spectrometer (Coradini et al. 2007). This experiment is composed of two sensor heads, -M (mapper) and $-\mathrm{H}$ (high resolution). The $-\mathrm{H}$ sensor is a highresolution punctual spectrometer mainly devoted to the study of the coma properties in the $1.88-5.03 \mu \mathrm{m}$ range, while $-\mathrm{M}$ produces hyperspectral images of the target in the $0.2-5.1 \mu \mathrm{m}$ range with an angular resolution of $250 \mu \mathrm{rad} \times 250 \mu \mathrm{rad}$. Data from VIRTIS-M are crucial to assess nucleus spectrophotometric properties: on a global scale with disk-integrated observations, and, thanks to its imaging capabilities, at higher spatial resolutions with disk-resolved measurements. The latter case is of paramount importance to map the compositional variability on the surface and possibly link it to sources of activity.

This work focuses on VIRTIS-M data acquired from July 2014 up to February 2015. The analysis we performed is divided into two main subjects: full-disk photometry (Sect. 2) and disk-resolved photometry (Sect. 3). In Sect. 2, we discuss rotational curves (Sect. 2.1), full-disk phase curves (Sect. 2.2), color ratios (Sect. 2.3) and the derivation of the geometric albedo (Sect. 2.4). In Sect. 3 we calculate a photometric reduction by means of a Hapke simplified model, taking advantage of the large dataset acquired by VIRTIS-M and assess the spectrophotometric properties of the surface. We also derived a zonal photometric reduction for four macro-regions to better investigate compositional and morphological differences on the nucleus 
(Sect. 3.6). In Sect. 4 the full-disk and the disk-resolved dataset are used together to derive a complete phase curve of the comet. A comparison to photometric properties of other comets is finally shown in Sect. 5, and in Sect. 6 we summarize the main findings and discuss future developments of this work with new observations in the coming months.

\section{Full-disk photometry}

In this section we analyze the photometric properties of $67 \mathrm{P}$ as observed by the VIRTIS instrument during the approach phase to the comet (MTP005 phase). Acquisitions have been taken on July 2014, when the comet was at an heliocentric distance of approximately 3.7 AU. The target-spacecraft distance decreased from $13000 \mathrm{~km}$ down to $1800 \mathrm{~km}$ in this period. As a consequence, the pixel dimension at the surface decreased from $3.2 \mathrm{~km}$ to $450 \mathrm{~m}$ and the portion of the VIRTIS field of view (FOV) occupied by the comet varied from a few pixels to up to a few tens. The whole dataset here and in the following sections was corrected for residual instrumental artifacts following the method described in Raponi (2015). Given the low spatial resolution of these acquisitions and the relatively low amount of pixels available for the analysis, a disk-resolved photometric study was not feasible, which justifed a disk-integrated approach. To this aim, the signal of the whole VIRTIS FOV was summed for each hyperspectral cube at each wavelength, producing a full-disk spectral reflectance. Because of the low activity of the comet, coma contribution to the total signal can be considered as negligible at the large heliocentric distance at that time. However, the average signal measured from background pixels was subtracted, which removed residual dark current contributions and minimized light scattered from the coma. Background pixels were selected as the closest to the comet disk image in the FOV, where the signal from the nucleus could not be recognized. Each acquisition is characterized by a value of the solar phase angle $\left(1.2^{\circ}<\alpha<14.9^{\circ}\right)$ and of the rotational phase. This allows us to produce both light curves and phase curves of the comet as shown below in Sects. 2.1 and 2.2.

\subsection{Light curves}

In Fig. 1 we report the light curves of the comet $67 \mathrm{P}$ as derived from VIRTIS MTP005 observations. Since the VIRTIS signal is calibrated into standard radiance units $\mathrm{W} / \mathrm{m}^{2} / \mu \mathrm{m} / \mathrm{sr}$ to be able to compare our result with previous measurements by Mottola et al. (2014), the derived flux $\left(F_{67 P, R},\left[\mathrm{~W} / \mathrm{m}^{2}\right]\right)$ was converted into reduced planetary magnitudes in the $R$-band $(R(1,1, \alpha))$ after convolution with the Kron-Cousins $R$-filter bandpass of (Bessell 1986) by means of the following relations (Lagerkvist \& Magnusson 1990):

$$
\begin{aligned}
& F_{67 P, R}=\Sigma_{i} I_{R, i} \delta \epsilon \\
& m_{R}=m_{\odot, R}-2.5 \log \frac{F_{67 P, R}}{F_{\odot, R}} \\
& R(1,1, \alpha)=m_{R}-5 \log r \cdot \Delta,
\end{aligned}
$$

where $I_{R, i}$ is the pixel radiance, $\delta \epsilon$ the instrumental instantaneous FOV (IFOV), $m_{R}$ the comet relative magnitude, $m_{\odot, R}$ the Sun relative magnitude, $F_{\odot, R}$ the solar flux at the comet heliocentric distance $r$, and $\Delta$ the spacecraft-comet distance. Both $r$ and $\Delta$ are expressed in astronomical units, while the subscript $R$ indicates that values refer to the $R$-band. Error bars are derived with the VIRTIS signal-to-noise ratio simulator described in

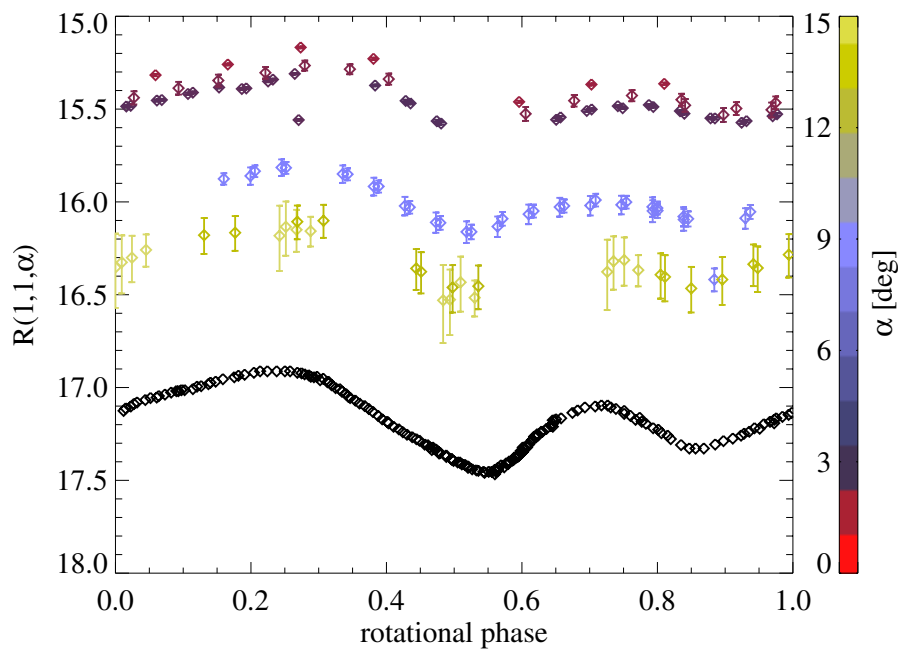

Fig. 1. Light curve in the $\mathrm{R}$ band. Black diamonds indicate the light curve as measured by the OSIRIS camera onboard Rosetta (Mottola et al. 2014), arbitrarily shifted to match the rotational phase of VIRTIS observations. We assumed a comet rotational period of $12.4043 \pm$ $0.0007 \mathrm{~h}$ (Sierks et al. 2015). The rotational phase is set to 0 at the time of the first VIRTIS acquisition $T_{0}=56850.0$ MJD (Modified Julian Date). The color bar provides the phase angle scale in degrees for VIRTIS observations.

Raponi (2015). In Fig. 1 points are grouped according to their phase angle $\alpha$. Because of the elongated irregular shape of 67P, these curves are double-peaked, with the primary maximum larger than the secondary. Moreover, they are shifted toward lower magnitudes for decreasing phase angles because a larger portion of the comet is both visible and illuminated and because of the opposition effect (OE) surge (Hapke et al. 1998). For comparison we report the light curve derived by the OSIRIS camera onboard Rosetta (Mottola et al. 2014) at $\alpha=32.6^{\circ}$, which shows that the results of the two instruments agree well.

\subsection{Low-phase integrated phase curves}

From the same dataset described in Sect. 2.2 we derived the phase curve of the comet, and we show it in Fig. 2 in four different wavelengths, $0.55 \mu \mathrm{m}, 0.80 \mu \mathrm{m}, 1 \mu \mathrm{m}$ and $2 \mu \mathrm{m}$. Data with similar phase angles but acquired at different rotational phases were averaged into $1^{\circ}$ phase angle bins. The phase curves were normalized at the lowest phase angle $\alpha=1^{\circ}$. The shape of the phase curve clearly displays a well-developed opposition effect $(\mathrm{OE})$ below $10^{\circ}$. No strong dependence on wavelength is observed, which indicates that shadow-hiding OE is the dominant mechanism (Hapke 1993), with coherent-backscattering OE possibly relevant only for phase angles lower than $1^{\circ}$ (Hapke 2002; Kaydash et al. 2013). A rigorous analytic description of the OE for full-disk observations can only be applied for a spherical body (Hapke 1993, 2012), which is not the case of comet $67 \mathrm{P}$. For this reason, we decided to postpone a full characterization of the OE parameters to a future work, when low phase angle disk-resolved observations will be available.

\subsection{Color ratios}

The same dataset as analyzed in Sects. 2.1 and 2.2 was used to compute color ratios at various wavelengths from integrated fluxes. Here we report three ratios as a function of the rotational phase (Fig. 3): one in the VIS range 
M. Ciarniello et al.: Photometric properties of comet 67P from VIRTIS-M aboard Rosetta

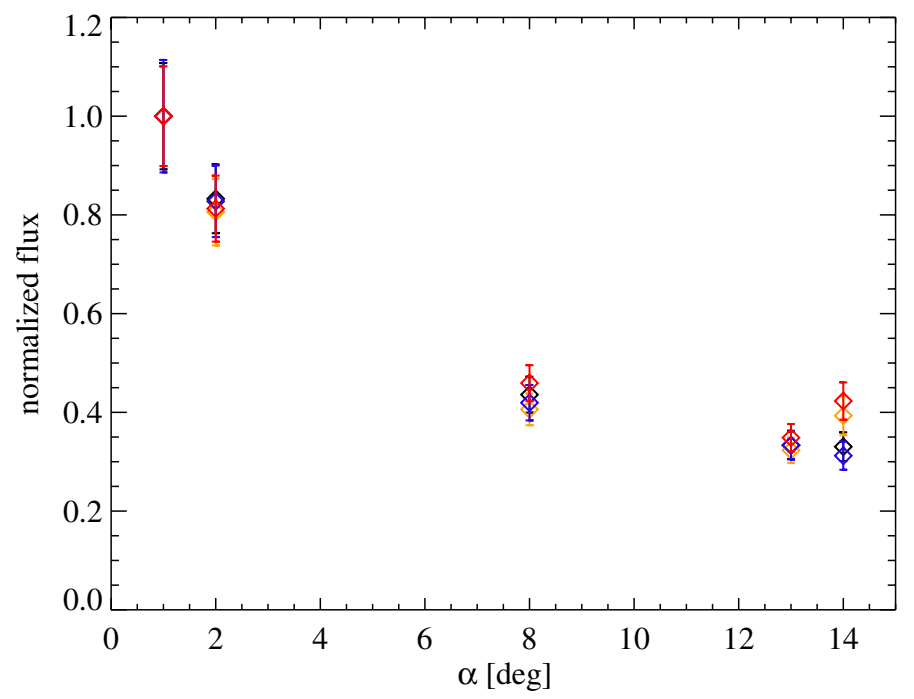

Fig. 2. Phase curve of the nucleus at low phase angles normalized at $1^{\circ}$. We report four different wavelengths: $0.55 \mu \mathrm{m}$ (black), $0.80 \mu \mathrm{m}$ (blue), $1 \mu \mathrm{m}$ (yellow), and $2 \mu \mathrm{m}$ (red). Error bars are from the flux variability that is due to the varying rotational phase.

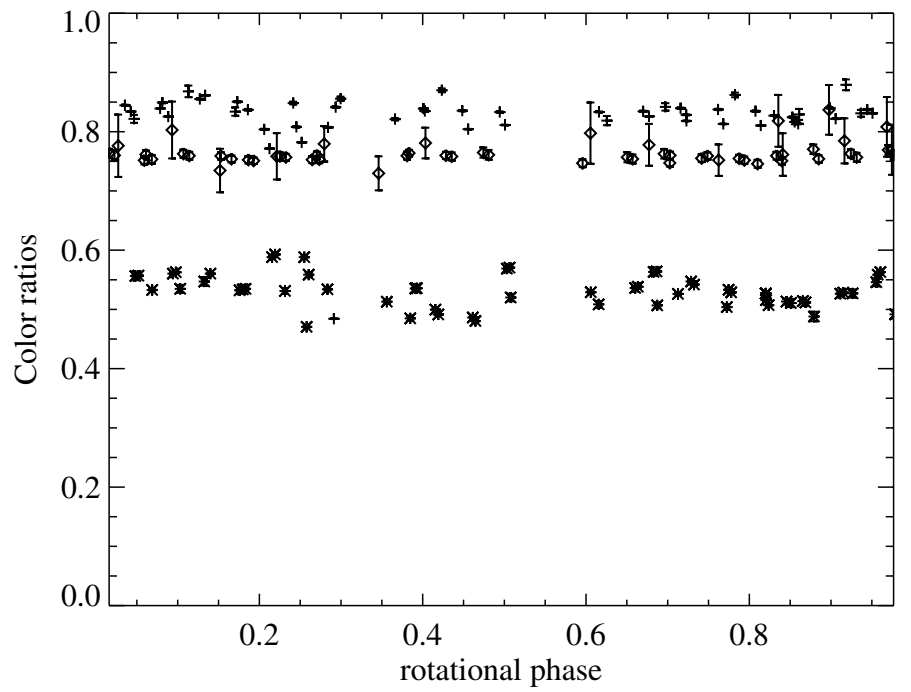

Fig. 3. Color ratios as a function of rotational phase. $C_{\mathrm{VIS}}$ is reported with diamonds, $C_{\mathrm{IR}}$ with crosses, and $C_{\mathrm{VIS}-\mathrm{IR}}$ with asterisks. Error bars are typically smaller than the symbol size, and the scatter of the points is due to the different solar phase angles of the individual acquisitions.

$\left(C_{\mathrm{VIS}}=\left(F_{0.55 \mu \mathrm{m}} / F_{0.8 \mu \mathrm{m}}\right) /\left(F_{\odot, 0.55} \mu \mathrm{m} / F_{\odot, 0.8} \mu \mathrm{m}\right)\right)$, one in the IR $\left(C_{\mathrm{IR}}=\left(F_{1 \mu \mathrm{m}} / F_{2 \mu \mathrm{m}}\right) /\left(F_{\odot, 1} \mu \mathrm{m} / F_{\odot, 2} \mu \mathrm{m}\right)\right)$, and one across the VIS and IR ranges $\left(C_{\text {VIS-IR }}=\left(F_{0.55 \mu \mathrm{m}} / F_{2 \mu \mathrm{m}}\right) /\left(F_{\odot, 0.55} \mu \mathrm{m} / F_{\odot, 2} \mu \mathrm{m}\right)\right)$. Acquisition with solar phase angles ranging in the $0^{\circ}-5^{\circ}$ interval were selected. $C_{\mathrm{VIS}}, C_{\mathrm{IR}}$ and $C_{\mathrm{VIS}-\mathrm{IR}}$ behave approximately as constants with no significant modulations. This indicates that surface spectral properties are fairly homogeneous on a global scale at the spatial resolution of the dataset.

\subsection{Geometric albedo}

In this section we derive the geometric albedo of $67 \mathrm{P}$ using the definition of normal albedo $A_{\mathrm{n}}$. Normal albedo is the reflectance of a surface observed at $0^{\circ}$ phase angle, normalized to the reflectance of a perfect Lambert surface at null incidence angle. This quantity is not an integrated value and can be straightforwardly computed from the reflectance of fully illuminated pixels

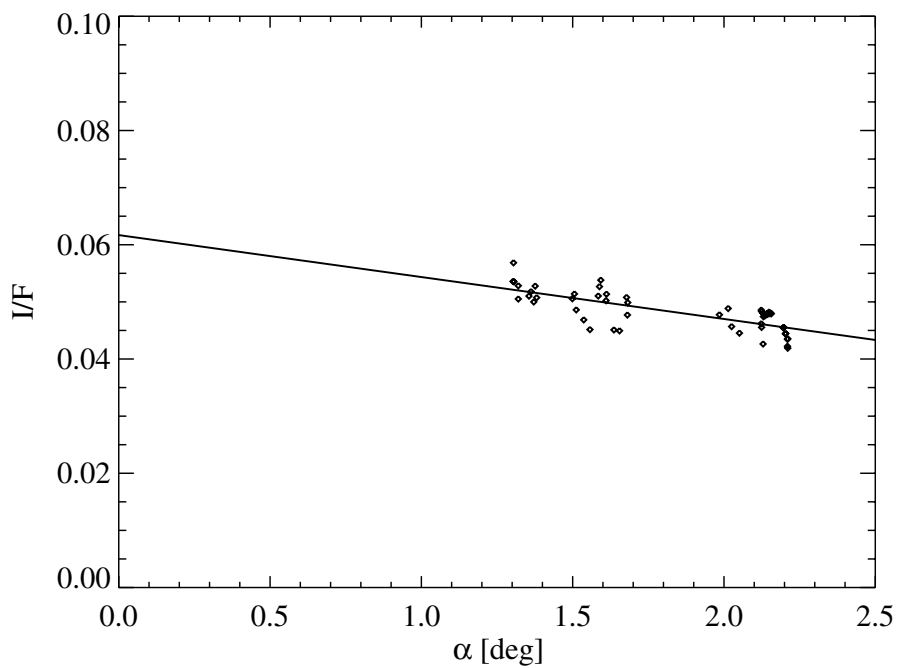

Fig. 4. Derivation of the normal albedo at $0.55 \mu \mathrm{m}$. The value at $0^{\circ}$ is extrapolated by means of a linear fit as shown in the plot.

Table 1. Geometric albedo of 67P as measured by VIRTIS compared to OSIRIS.

\begin{tabular}{ccc}
\hline \hline Wavelength $[\mu \mathrm{m}]$ & $A_{\text {geo }}$ (this work) & $A_{\text {geo }}$ (Fornasier et al. 2015) \\
\hline 0.480 & $0.054 \pm 0.002$ & $0.0554 \pm 0.0024$ \\
0.535 & $0.059 \pm 0.002$ & $0.0589 \pm 0.0034$ \\
$0.649^{a}$ & $0.074 \pm 0.002$ & $0.0677 \pm 0.0039$ \\
0.700 & $0.077 \pm 0.002$ & $0.0720 \pm 0.0031$ \\
0.743 & $0.080 \pm 0.003$ & $0.0766 \pm 0.0031$ \\
\hline
\end{tabular}

Notes. ${ }^{(a)}$ The value at $0.649 \mu \mathrm{m}$ for the VIRTIS case is computed as an interpolation on the local continuum after removing the wavelengths in the $0.642-0.653 \mu \mathrm{m}$ range, which are affected by a junction of the instrument spectral filters.

acquired at null $\alpha$. From the whole MTP005 observations dataset we selected pixels at the lowest phase angles $\left(1^{\circ}-2.5^{\circ}\right)$ that were fully illuminated (incidence and emission angles below $10^{\circ}$ ), as shown in Fig. 4. An approximated linear trend of the radiance factor $(I / F)$ with phase angle can be recognized, and we extrapolated the value at $\alpha=0^{\circ}$ by means of a linear fit. The value obtained at $0.55 \mu \mathrm{m}$ is $A_{\mathrm{n}}=0.062 \pm 0.002$, which refines the value previously reported in Capaccioni et al. (2015). In the approximation of single scattering, which can be safely applied to the case of a dark object like 67P, the normal albedo has the same expression as the geometric albedo (Hapke 2012), the latter being the average value of the former over the illuminated portion of the target body. For this reason we assume that the value of $A_{\mathrm{n}}$ derived in this section matches the geometric albedo $A_{\text {geo }}$ of the comet. To compare our results to those derived in Fornasier et al. (2015), we derived the geometric albedo at wavelengths corresponding to OSIRIS' filters bandpasses with the same technique as described in this section. Because of the larger noise affecting wavelengths shortward of $0.4 \mu \mathrm{m}$ and the presence of a spectral artifact in $0.8-1.0 \mu \mathrm{m}$ (see Sect. 3.1.2), we limited the comparison to five wavelengths in the $0.4-0.8 \mu \mathrm{m}$ range, as reported in Table 1 . The results of the two instruments agree well.

\section{Disk-resolved photometry}

In this section we analyze the data acquired during MTP006 to MTP12 sequences. The distance of the nucleus of $67 \mathrm{P}$ to the spacecraft varies between $384 \mathrm{~km}$ and $7.7 \mathrm{~km}$, providing a 


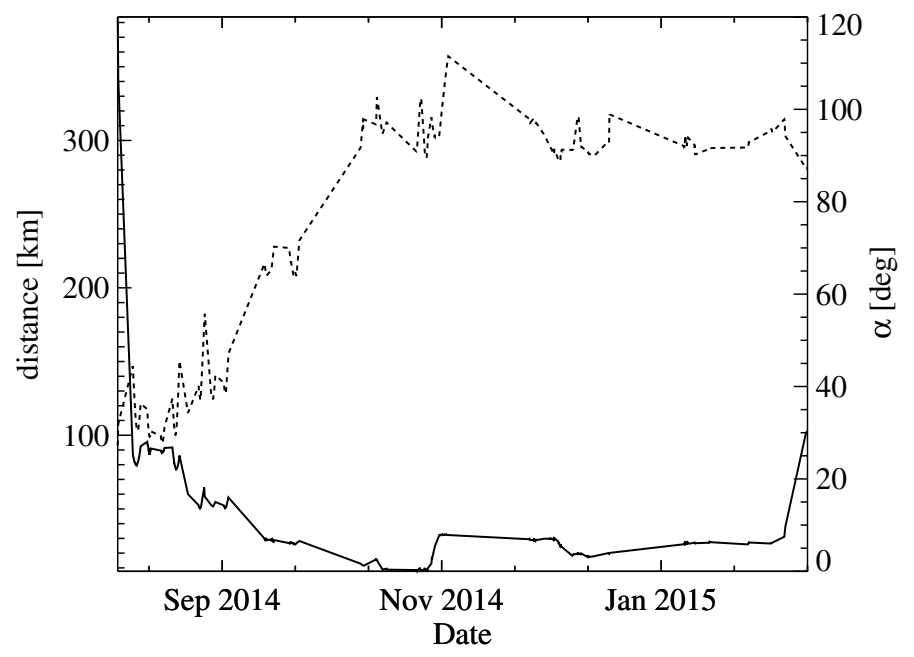

Fig. 5. Average target-spacecraft distance (solid line) and phase angle (dashed line) for the different VIRTIS-M acquisitions.

spatial resolution from $96 \mathrm{~m}$ down to $1.9 \mathrm{~m}$ (see Fig. 5), with most of the observations taken at a resolution below $25 \mathrm{~m} /$ pixel. The phase angle ranges in an interval of $27.2^{\circ}-111.5^{\circ}$ (see Fig. 5), and different regions were observed several times under varying observation geometries. To compare data acquired under different illumination conditions, it is necessary to separate photometric effects from the spectral properties of the surface. This requires the photometric reduction of the signal measured by VIRTIS to a quantity that is linked to the intrinsic brightness of the surface.

\subsection{Photometric reduction}

To compute a photometric reduction, we require a model that links the reflectance of a particulate medium to the observation geometries and physical properties of the surface. There are several models that accomplish this task, based on empirical, semi-empirical, or physical assumptions (Akimov 1988; Longobardo et al. 2014; Minnaert 1941; Shkuratov et al. 1999). We here adopted the well-known Hapke model (Hapke 1993, 2002, 2012). This solution of the radiative transfer equation has been extensively applied in planetary science to interpret data from remote-sensing measurements (Buratti et al. 2004; Ciarniello et al. 2011; Domingue et al. 1995), and in particular to investigate photometric properties of cometary nuclei ( $\mathrm{Li}$ et al. 2007b,a, 2009, 2013). The expression of the bidirectional reflectance of a semi-infinite medium from Hapke's model is given by the following equation:

$$
\begin{aligned}
& r(i, e, \alpha)=\frac{w}{4 \pi} \frac{\mu_{0 \mathrm{eff}}}{\mu_{0 \mathrm{eff}}+\mu_{\mathrm{eff}}} \\
& \times\left[B_{\mathrm{SH}}(\alpha) p(\alpha)+H\left(w, \mu_{0 \mathrm{eff}}\right) H\left(w, \mu_{\mathrm{eff}}\right)-1\right] \times S(i, e, \alpha, \bar{\theta}) B_{\mathrm{CB}}(\alpha),
\end{aligned}
$$

where

- i,e, $\alpha$ are incidence, emission, and phase angle;

- $w$ is the single scattering albedo (SSA);

- $p(\alpha)$ is the single particle phase function (SPPF);

- $\mu_{0 \mathrm{eff}}, \mu_{\mathrm{eff}}$ are effective cosines of incidence and emission angle, respectively;

- $H(w, x)$ are the Chandrasekhar functions;

- $B_{\mathrm{SH}}$ is the shadow-hiding opposition effect (SHOE);
- $B_{\mathrm{CB}}$ is the coherent-backscattering opposition effect (CBOE);

- $S(i, e, \alpha, \bar{\theta})$ is the shadowing function for large-scale roughness;

- $\bar{\theta}$ is the average surface slope.

We refer to Hapke (1993) for a complete description of every single term of Eq. (2). Considering the lack of observations at low phase angles for the disk-resolved dataset $\left(27.2^{\circ}<\alpha<111.5^{\circ}\right)$ and the extremely low albedo of comet surface (see Sect. 2.4), the formulation given in Eq. (2) can be simplified posing to 1 the opposition effect terms $B_{\mathrm{SH}}, B_{\mathrm{CB}}$, and the function $H(w, x)$ that describes multiple scattering (single scattering approximation). Equation (2) is then reduced to

$r(i, e, \alpha)=\frac{I / F}{\pi}=\frac{w}{4 \pi} \frac{\mu_{0 \mathrm{eff}}}{\mu_{0 \mathrm{eff}}+\mu_{\mathrm{eff}}} p(\alpha) S(i, e, \alpha, \bar{\theta})$,

where three quantities link the reflectance to the spectrophotometric properties of the surface: $w, p(\alpha)$, and $\bar{\theta}$. The SPPF is modeled following Heyney \& Greenstein (1941),

$p(\alpha)=\frac{1-b^{2}}{\left(1+2 b \cos (\alpha)+b^{2}\right)^{3 / 2}}$,

where $b$ is the asymmetry parameter that determines the SPPF behavior:

$$
\begin{aligned}
& -b<0 \text { : backscattering } \\
& -b>0 \text { : forward-scattering. }
\end{aligned}
$$

The advantage of the formulation in Eq. (3) is that in contrast to Eq. (2), the photometric reduction is achieved by means of a multiplicative term, thereby enabling the SSA to be computed in closed form once the model parameters are determined. However, it must be pointed out that neglecting the contribution of multiple scattering in the modeling might introduce a limited overestimation of the retrieved $w$, while the elimination of the OE term, which should be negligible in the phase angle range used, might in principle be compensated for by an adjustment of $w, b$. We note that a refinement of the Hapke model that accounts for the effect of porosity has been developed in Hapke $(2008,2012)$ and was discussed in Ciarniello et al. (2014). Nevertheless, the vast majority of the photometric studies that use Hapke's theory applied the formulation given in Eq. (2), which is the one we used here to be able to provide a direct comparison of our results with those derived in previous analyses. Moreover, the effect of porosity cannot be separated from SSA if multiple scattering is negligible (Li et al. 2013) and OE is excluded, thus making the application of Hapke (2008) model impractical for this work.

The whole dataset we analyzed to derive the photometric parameters was filtered to avoid unfavorable observation geometries that are due to large incidence and emission angles, imposing $i, e<60^{\circ}$ and pixels with extremely low reflectance $(\mathrm{I} / \mathrm{F}<0.001$ at $0.55 \mu \mathrm{m})$. In particular, this allow us to sort out pixels that are dominated by noise and minimize the number of residual partly shadowed pixels that are not ruled out using the adopted shape model of the nucleus from Preusked et al. (2015) and to limit the effect induced by the error on the geometry. For the latter point we recall that the relative error on $\cos (i)$ and $\cos (e)$ diverges when $i, e \rightarrow 90^{\circ}$. This choice fixes the number of analyzed pixels to more than $2,100,000$. This represents a statistically representative sample of the global surface properties of the nucleus, also preserving acquisitions at large 


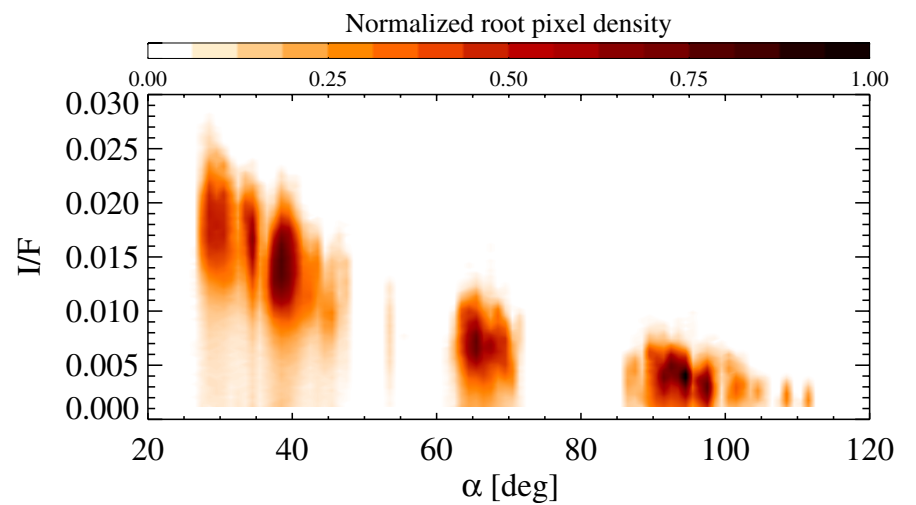

Fig. 6. $I / F$ at $0.55 \mu \mathrm{m}$ as a function of the phase angle: contour plot showing the pixel density.

phase angle, as shown in Fig. 6, where the pixel density is reported in a $I / F$ vs. $\alpha$ plot. The distribution develops along a decreasing monotonic curve that links reflectance to phase angle, as expected. Photometric reduction was computed under the assumption that the photometric properties of the comet surface are globally homogeneous at the spatial resolution of the VIRTIS observations. This implies that $b$ and $\bar{\theta}$ are constant over the surface. As shown below, in deriving $b$ and $\bar{\theta}$, we determine a global spectrum of $w$. This quantity can be considered as an average SSA spectrum of the comet. However, we assumed that most of the intrinsic brightness variability of comet surface is contained in the SSA. This allows, as a further step, using the derived $b$ and $\bar{\theta}$ in Eq. (3) to independently estimate a value of $w$ from each pixel, thus mapping the variation of albedo across the nucleus surface and to compare observations of the same regions acquired at different times along the comet orbit during the Rosetta mission.

To derive the set of Hapke parameters, we applied the following iterative algorithm:

- The $I / F$ at a given wavelength of each pixel is multiplied by the factor $4 \frac{\mu_{0 \text { eff }}+\mu_{\text {eff }}}{\mu_{0 \text { eff }} S\left(i, e, \alpha, \overline{\theta_{0}}\right)}$, where $\overline{\theta_{0}}$ is a given value of the roughness slope parameter. Assuming Eq. (3), this quantity represents $w p(\alpha)$, and we derive a distribution like the one shown in Fig. 7.

- The distribution of $w p(\alpha)$ is averaged in $1^{\circ}$ phase angle bins.

- The average curve of $w p(\alpha)$ is fitted, thus retrieving $b$ and $w$.

- The same procedure is repeated for all the VIRTIS wavelengths: residuals from selected wavelengths across VIS-IR are summed.

- The whole algorithm is repeated for $\overline{\theta_{0}}$ varying in the $\left[1^{\circ}-60^{\circ}\right]$ range with $1^{\circ}$ steps (Fig. 8).

- The final $w, b, \bar{\theta}$ are selected as those that provide the lowest residuals.

We recall that $w$ and $b$ in general are wavelength-dependent quantities, while $\bar{\theta}$ is not, since it is linked to the morphology of the surface.

\subsubsection{Surface roughness and $\bar{\theta}$}

In Fig. 9 we report the total residuals of the fitting procedure described in Sect. 3.1 as a function of $\bar{\theta}$. We computed this quantity with the equation

$\operatorname{Res}(\bar{\theta})=\sum_{\lambda=0.55}^{2 \mu \mathrm{m}} \chi_{\lambda}^{2}(\bar{\theta})$,

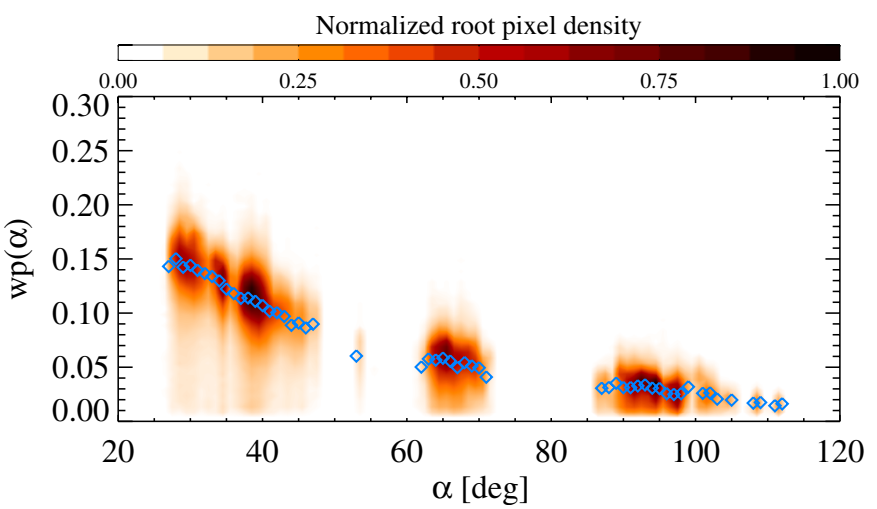

Fig. 7. $w p(\alpha)$ distribution at $0.55 \mu \mathrm{m}$ as a function of phase angle for $\bar{\theta}_{0}=0^{\circ}$. Blue diamonds represent the average values on $1^{\circ}$ phase angle bins.

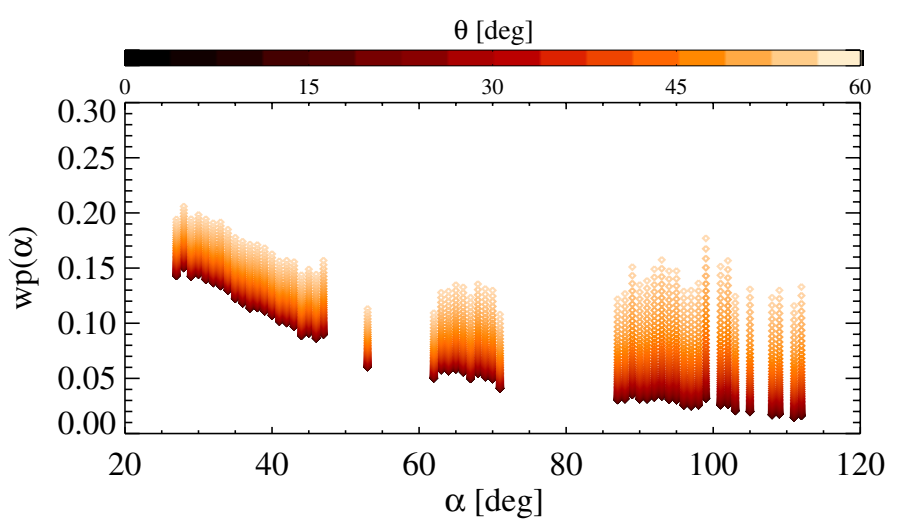

Fig. 8. $w p(\alpha)$ averaged on $1^{\circ}$ phase angle bins for $\bar{\theta}$ varying in the $1^{\circ}-60^{\circ}$ range. The color bar provides $\bar{\theta}$ values. Increasing roughness affects the estimated $w p(\alpha)$, which induces a flatter distribution with a higher overall level.

where $\chi_{\lambda}^{2}(\bar{\theta})$ is the resulting chi-square value of the fit of the average $w p(\alpha)$ curve derived assuming $\bar{\theta}$, at wavelength $\lambda$ (Fig. 8). Residuals were summed in the $0.55-2 \mu \mathrm{m}$ range to exclude both shorter wavelengths, which are affected by higher noise, and longer ones, where thermal emission can partially be present even if it is not dominant. The lowest value of the residuals is obtained at $\bar{\theta}=19^{\circ}$. The physical meaning of the surface slope parameter is still debated. While commonly related to the shadowing due to large-scale roughness (Hapke 2012), laboratory studies from Shepard \& Helfenstein (2011) indicated that it can be affected by submm-scale structures. Moreover, compensation effects with other photometric parameters in the fit procedure, such as the asymmetry factor of SPPF and SSA, can also modify the retrieved value of $\bar{\theta}$, and this must be taken into account when interpreting its meaning. This effect could be strong when there are no observations at very large phase angles. Apart from these problems, we can estimate the error on $\bar{\theta}$ by examining the curve in Fig. 9: the level of the first part of the curve is not sensitive to the slope parameter and then it was assumed as a sensitivity threshold to the effects of the roughness, thus obtaining a final value of $\bar{\theta}=19_{-9}^{\circ+4}$.

\subsubsection{SSA}

In Fig. 10 we report the derived spectrum of the SSA. As shown previously in Capaccioni et al. (2015), the spectrum of the comet surface is reddish, with a steeper slope in the VIS region. The 


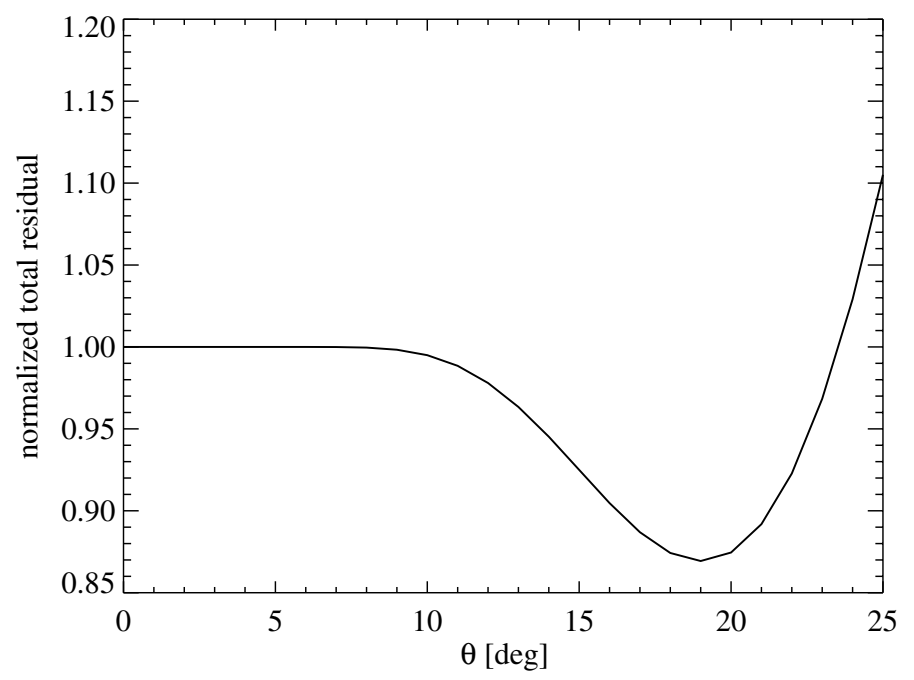

Fig. 9. Total residual as a function of the roughness slope parameter $\bar{\theta}$ normalized at $\bar{\theta}=0^{\circ}$.

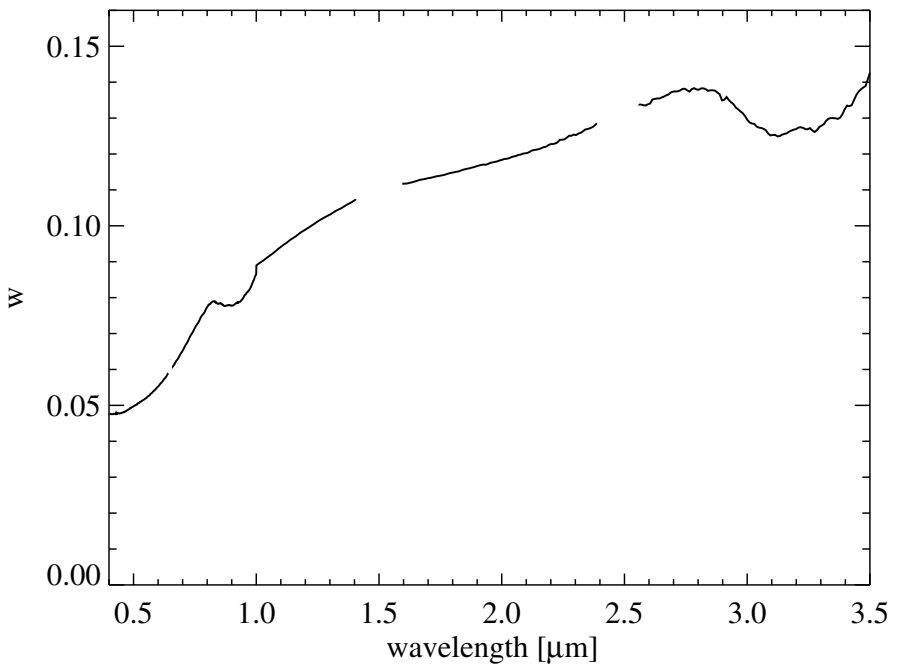

Fig. 10. Spectral variation of the SSA $w$ as derived from the computation of the photometric parameters. Missing parts of the spectrum correspond to junctions of the instrumental spectral filters. The absorption in the $0.8-1 \mu \mathrm{m}$ is a residual artifact from calibration.

overall level of $w$ is extremely low, with a value of 0.052 at $0.55 \mu \mathrm{m}$ that increases in the IR to 0.14 at $2.8 \mu \mathrm{m}$. The $3.2 \mu \mathrm{m}$ absorption feature, caused by the presence of organic material, is clearly visible up to $3.5 \mu \mathrm{m}$, where the thermal emission starts to affect the spectrum. A feature centered at $0.9 \mu \mathrm{m}$ can be noted, but it is currently interpreted as a residual artifact from calibration.

As stated above, the derived SSA can be considered as representative of the average properties of the surface of 67P. However, spatial albedo variability is expected at the VIRTIS observation scale, and this is the information we aim to extract after applying the photometric reduction as shown in Sect. 3.3.

\subsubsection{SPPF}

In Fig. 11 we report the spectrum of the derived asymmetry factor $b$. The value at $0.55 \mu \mathrm{m}$ is $b=-0.42$, indicating a strong backscattering behavior, as observed for other comets (Table 3), slightly increasing with wavelength. As stated above, the asymmetry factor drives the behavior of the phase curve. The phase

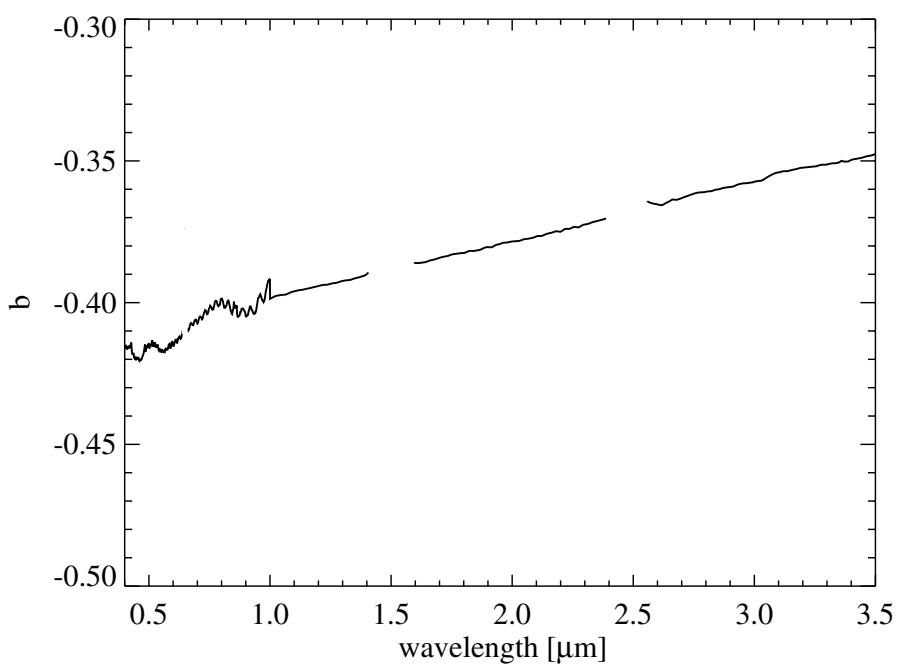

Fig. 11. Spectral variation of the asymmetry factor $b$ as derived from the computation of the photometric parameters. Missing parts of the spectrum correspond to junctions of the instrumental spectral filters.

Table 2. Hapke modeling of the photometric properties of the nucleus of $67 \mathrm{P}$ as derived from VIRTIS and OSIRIS data at $0.65 \mu \mathrm{m}$.

\begin{tabular}{cccccccc}
\hline \hline Instrument & $B_{0 \mathrm{SH}}$ & $h_{\mathrm{SH}}$ & $B_{0 \mathrm{CB}}$ & $h_{\mathrm{CB}}$ & $b$ & $w$ & $\bar{\theta}$ \\
\hline VIRTIS (this work) $^{a}$ & - & - & - & - & -0.41 & 0.060 & $19^{\circ}$ \\
OSIRIS & 2.5 & 0.079 & 0.188 & 0.017 & -0.37 & 0.042 & $15^{\circ}$ \\
\hline
\end{tabular}

Notes. ${ }^{(a)}$ The values are computed as an interpolation on the local continuum after removing the wavelengths in the $0.642-0.653 \mu \mathrm{m}$ range, which are affected by a junction of the instrument spectral filters.

angle range investigated in this work, albeit limited, can be considered wide enough to provide a robust determination of $b$ since it covers the part of the phase curve that is most sensitive to this parameter. In fact, at very small and very large phase angles, the opposition effect and large-scale surface roughness dominate, respectively; this masks the effect of the SPPF.

\subsection{Comparison with Hapke modeling of OSIRIS data}

Fornasier et al. (2015) have applied Hapke modeling to images of the nucleus of 67P acquired by the OSIRIS Narrow Angle Camera onboard Rosetta. As a result of the better spatial resolution with respect to VIRTIS (18.6 $\mu \mathrm{rad} /$ pix, Keller et al. 2007), the OSIRIS camera produced disk-resolved images of the nucleus of 67P during the MTP005 phase, with solar phase angles down to $1.3^{\circ}$. This enabled Fornasier et al. (2015) to characterize the opposition effect region of the phase curve as well by determining the SHOE amplitude $B_{0 \mathrm{SH}}$ and width $h_{\mathrm{SH}}$ and analog parameters for the CBOE $\left(B_{0 \mathrm{CB}}\right.$ and $\left.h_{\mathrm{CB}}\right)$. Two different Hapke models have been adopted, described in Hapke (2002, 2012), respectively, the latter including the effect of porosity. Here we compare our Hapke modeling results with those obtained by OSIRIS for the case reported in Hapke (2002), which is more similar to our approach. The results are listed in Table 2 .

The two studies converge on a low SSA and a backscattering behavior of the SPPF. However, it must be noted that we here obtain a higher value of the SSA and of the absolute value of $b$. This discrepancy is explained by the different versions of the Hapke model that were adopted. In particular, the inclusion of the $\mathrm{OE}$ in the model, which was necessary in Fornasier et al. (2015) to describe low phase angle observations, can also account for 
Table 3. Spectrophotometric properties and Hapke parameters of 67P compared to other cometary nuclei.

\begin{tabular}{|c|c|c|c|c|c|c|c|c|c|}
\hline Comet & $B-V$ & $V-R$ & $R-I$ & SSA & $b$ & $\bar{\theta}$ & $A_{\text {geo }}$ & $\beta\left[\mathrm{mag} /{ }^{\circ}\right]$ & Phase angle coverage \\
\hline $67 \mathrm{P}^{a}$ & $0.73 \pm 0.07$ & $0.57 \pm 0.03$ & $0.59 \pm 0.04$ & $0.052 \pm 0.013$ & -0.42 & $19^{\circ+4}$ & $0.062 \pm 0.002$ & $0.077 \pm 0.002^{b}, 0.041 \pm 0.001^{c}$ & $1.2^{\circ}-111.5^{\circ}$ \\
\hline $67 \mathrm{P}^{d}$ & $\begin{array}{c}0.13 \pm 0.07 \\
-\end{array}$ & $\begin{array}{c}0.01+0.03 \\
-\end{array}$ & $\begin{array}{c}0.03 \pm 0.04 \\
-\end{array}$ & $0.037 \pm 0.002$ & $-0.42 \pm 0.03$ & $15^{-9}$ & $0.059 \pm 0.02^{e}$ & $0.047 \pm 0.002$ & $1.3^{\circ}-53.9^{\circ}$ \\
\hline $67 \mathrm{P}^{f}$ & - & $0.52 \pm 0.05$ & - & - & - & - & - & - & $4.8^{\circ}$ \\
\hline $67 \mathrm{P}^{g}$ & $0.83 \pm 0.08$ & $0.54 \pm 0.05$ & $0.46 \pm 0.04$ & - & - & - & - & $0.061-0.076$ & $0.5^{\circ}-10.6^{\circ}$ \\
\hline 1P/Halley ${ }^{h}$ & $0.72 \pm 0.04$ & $0.41 \pm 0.03$ & $0.39 \pm 0.06$ & - & - & - & - & $\begin{array}{c}0.001-0.070 \\
-\end{array}$ & $107^{\circ}$ \\
\hline 19P/Borrelly ${ }^{i}$ & - & - & - & $0.057 \pm 0.009$ & $-0.43 \pm 0.07$ & $22^{\circ} \pm 5^{\circ}$ & $0.072 \pm 0.020$ & 0.043 & $51^{\circ}-75^{\circ}$ \\
\hline 19P/Borrelly ${ }^{l}$ & - & $0.25 \pm 0.78$ & - & $\begin{array}{c}0.001 \pm 0.003 \\
-\end{array}$ & $\begin{array}{c}-0.45 \pm 0.07 \\
-\end{array}$ & $\begin{array}{c}22 \\
-\end{array}$ & $\begin{array}{c}0.012 \pm 0.020 \\
-\end{array}$ & - & $1.98^{\circ}$ \\
\hline $103 \mathrm{P} /$ Hartley $2^{m}$ & $0.75 \pm 0.05$ & $0.43 \pm 0.04$ & - & $0.036 \pm 0.006$ & $-0.46 \pm 0.06$ & $15^{\circ} \pm 10^{\circ}$ & $0.045 \pm 0.009$ & $0.046 \pm 0.002$ & $79^{\circ}-93^{\circ}$ \\
\hline 9P/Tempel $1^{n}$ & $0.84 \pm 0.01$ & $0.50 \pm 0.01$ & $0.49 \pm 0.02$ & $0.039 \pm 0.005$ & $-0.49 \pm 0.02$ & $16^{\circ} \pm 8^{\circ}$ & $0.051 \pm 0.009$ & $0.046 \pm 0.007$ & $63^{\circ}-117^{\circ}$ \\
\hline $81 / \mathrm{P}$ Wild $2^{o}$ & - & - & - & $0.038 \pm 0.04$ & $-0.52 \pm 0.04$ & $27 \pm 5^{\circ}$ & 0.059 & $0.0513 \pm 0.0002$ & $11^{\circ}-100^{\circ}$ \\
\hline
\end{tabular}

Notes. ${ }^{(a)}$ This work. ${ }^{(b)}$ For $\alpha<15^{\circ} .{ }^{(c)}$ For $\alpha>15^{\circ} .{ }^{(d)} w, b$ (at $\left.0.535 \mu \mathrm{m}\right), \bar{\theta}$ and $\beta$ from Fornasier et al. (2015). ${ }^{(e)}$ Sierks et al. (2015). ${ }^{(f)}$ Lamy et al. (2006). ${ }^{\left({ }^{g}\right)}$ Tubiana et al. (2011). $B-V$ has been computed from $B-R$ and $V-R$ measurements reported in Tubiana et al. (2011). ${ }^{(h)}$ Thomas $\&$ Keller (1989). ${ }^{(i)}$ Photometric parameters derived in $R$-band by Li et al. (2007b). Ground-based observations with solar phase angle down to $13^{\circ}$ have been included to compute the slope parameter. ${ }^{(l)}$ Lowry et al. (2003). ${ }^{(m)}$ Li et al. (2013). Gemini and HST observations at low phase angle have been included to constrain Hapke modeling and derive the slope parameter. ${ }^{(n)}$ Photometric parameters derived in $V$-band Li et al. (2007a). Ground-based and HST observations with solar phase angle down to $4^{\circ}$ have been included to compute the slope parameter. ${ }^{(o)}$ Photometric parameters derived in $R$-band by Li et al. (2009).

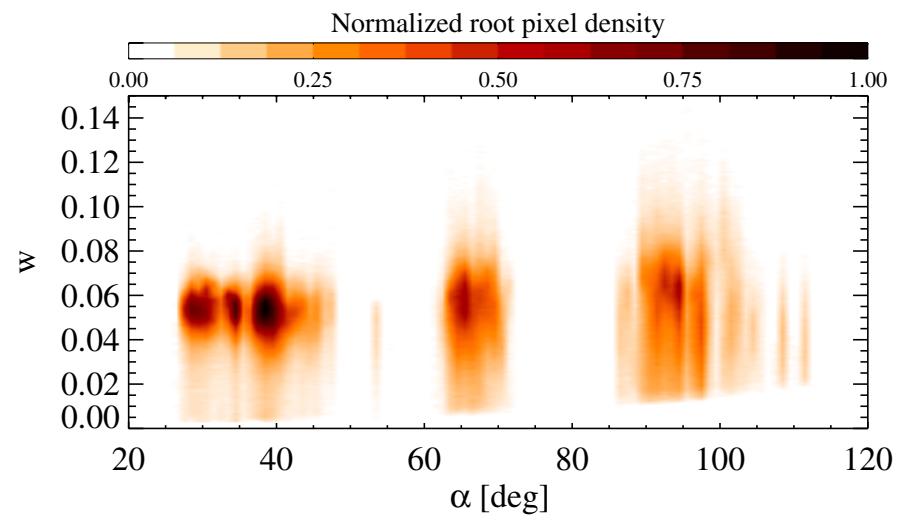

Fig. 12. SSA at $0.55 \mu \mathrm{m}$ vs. phase angle. The color bar indicates the root pixel density normalized at the highest value. The correlation coefficient is $R_{w, \mathrm{~g}}=0.03$.

part of the photometric output at relatively large phase angles $\left(>20^{\circ}\right)$, therefore it requires a smaller SSA and a less backscattering SPPF, as in the OSIRIS case. This effect can be strong for very high values of $B_{0 S H}(>1)$, where, in the case of backscattering surfaces, the SHOE term on one side and the SPPF and the SSA on the other can compensate for one another. This makes a clear distinction between the two solutions difficult. In this context, CBOE plays a minor role because it has smaller amplitude and angular width. We also retrieve a somewhat higher value of about $25 \%$ of the roughness slope parameter $\bar{\theta}$ than did Fornasier et al. (2015), which is because our dataset extends to larger solar phase angles (up to $111.5^{\circ}$ ) than the OSIRIS images (up to $53.9^{\circ}$ ). This makes it more sensitive to the effect of subpixel roughness.

\subsection{Accuracy of the photometric reduction and photometrically reduced data}

After the photometric parameters $(b, \bar{\theta})$ have been retrieved, they can be applied to invert Eq. (3) to derive the SSA from the $I / F$ measured by VIRTIS for each acquired pixel on the surface. In Fig. 12 we report the derived SSA at $0.55 \mu \mathrm{m}$ after applying the photometric reduction to all the pixels of Fig. 6. This effectively removes the dependence on phase angle, as shown by the very low correlation coefficient $\left(R_{w, \mathrm{~g}}=0.03\right)$, and the cloud of values is distributed around the global value obtained in Sect. 3.1.2

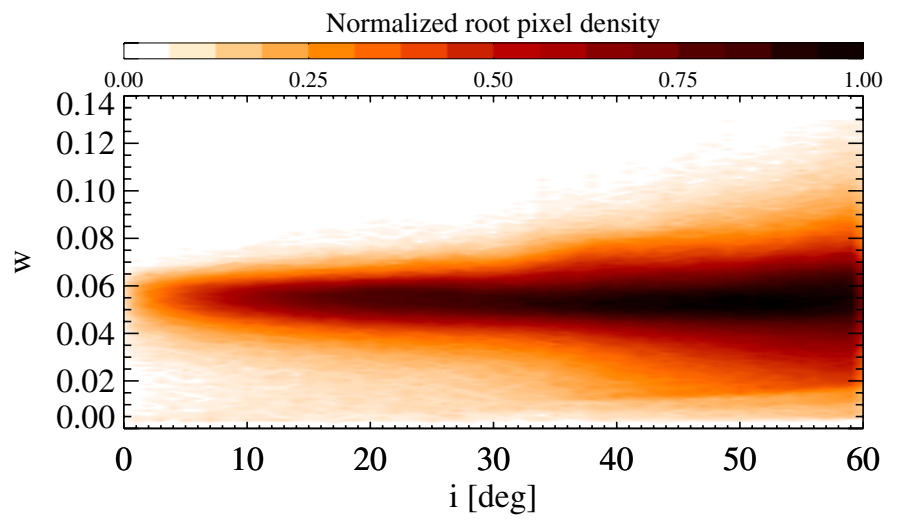

(a)

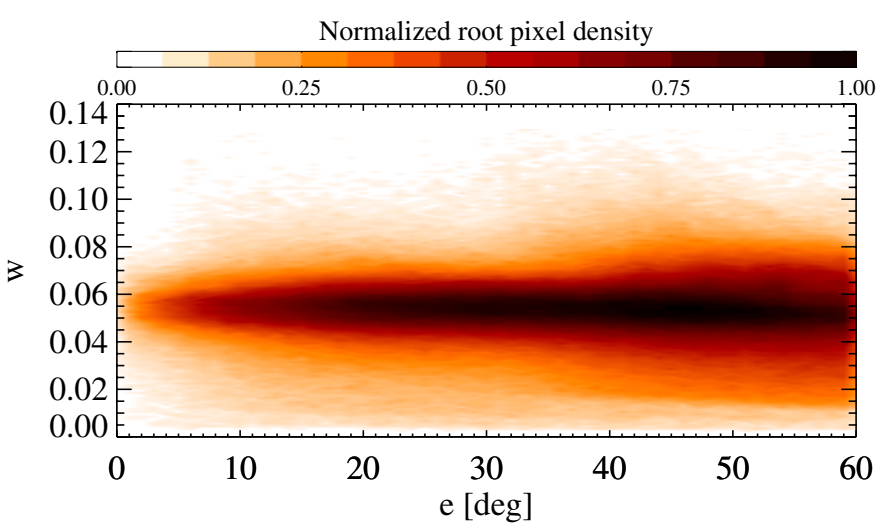

(b)

Fig. 13. SSA at $0.55 \mu \mathrm{m}$ vs. incidence angle a) and emission angle b). The color bar indicates the root pixel density normalized at the highest value. The correlation coefficients are $R_{w, i}=0.03$ and $R_{w, e}=-0.05$.

$(w=0.052)$. The width of the distribution at each phase angle is related to the intrinsic variability of the surface properties, to measurement errors as well as to possible compensating effects generated by assuming a single determination of $b$ and $\bar{\theta}$ for the entire surface.

In Fig. 13 we report the derived SSA values at $0.55 \mu \mathrm{m}$ as a function of the incidence and emission angles. In both cases the SSA is substantially independent of $i$ and $e$, with $R_{w, i}=0.03$ and $R_{w, e}=-0.05$, respectively. 


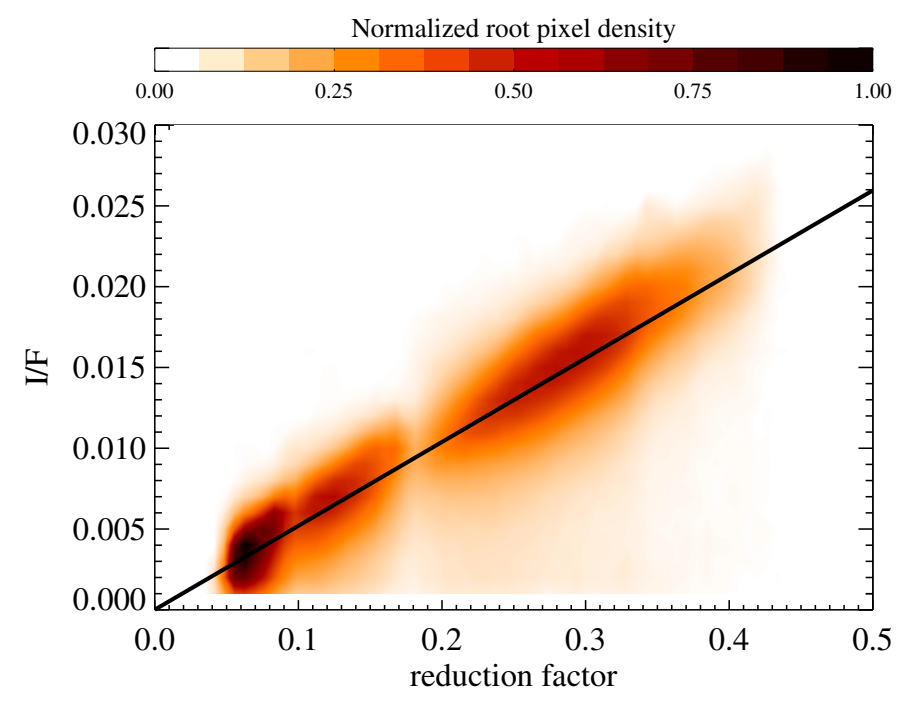

Fig. 14. $I / F$ at $0.55 \mu \mathrm{m}$ vs. the reduction factor. The color bar indicates the root pixel density normalized at the highest value. The linear fit is plotted as a black line with equation $I / F=0.052 \times r f+0.000033$.

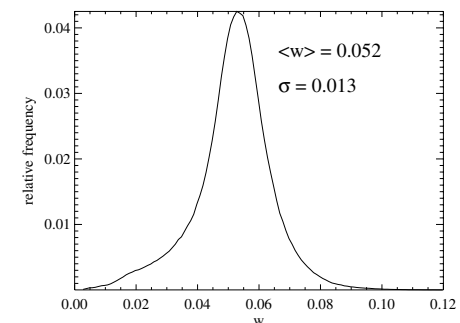

(a)

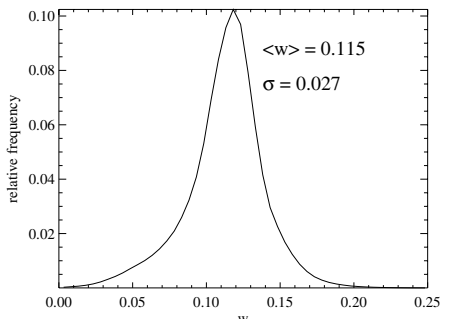

(b)
Fig. 15. Histograms of the derived SSA at $0.55 \mu \mathrm{m}$ (left) and $1.8 \mu \mathrm{m}$ $($ right). Both curves have been normalized at the total number of pixels. Mean value and standard deviation are indicated in the plots.

To further check the accuracy of the derived photometric reduction, we compared the $I / F$ as measured by VIRTIS with the reduction factor $r f$ :

$r f=\frac{\mu_{0 \mathrm{eff}}}{\mu_{0 \mathrm{eff}}+\mu_{\mathrm{eff}}} \frac{p(\alpha)}{4} S(i, e, \alpha, \bar{\theta})$.

As shown in Fig. 14, these two quantities show a linear correlation that intercepts the origin, indicating that the photometric model we applied is appropriate to describe the reflectance of the surface of the nucleus. In particular, the fitted slope coefficient is 0.052 , and this quantity can be interpreted as the average SSA of the surface, confirming the value shown in Fig. 10 at $0.55 \mu \mathrm{m}$. From Fig. 14 it can be noted that no points with peculiar photometric properties can be isolated, indicating a certain homogeneity of the surface. This is confirmed in Fig. 15, where we show histograms of the derived $w$ values at $0.55 \mu \mathrm{m}$ and $1.8 \mu \mathrm{m}$. The two distributions are unimodal, meaning that we cannot recognize terrains with a statistically different behavior at the VIRTIS pixel scale. Only an elongated tail toward low values of $w$ can be found in both distributions; this might be related to regions with lower albedo (see Sect. 3.5) or residual partly shadowed pixels.

In Fig. 16 we show the result of the photometric reduction at $0.55 \mu \mathrm{m}$ on typical VIRTIS hyper-spectral cubes. Bright regions in $I / F$ images disappear in the photometrically reduced ones, revealing that the SSA is similar to that of the surrounding terrains. However, some residuals of the topography can still be recognized in the photometrically reduced cubes. This is due to the presence of shadows and a miscorrection in correspondence of sharp reliefs, where the incidence and emission angle can be either large or can abruptly vary at subpixel scale.

\subsection{Spectral slopes and phase reddening}

In Figs. 17a and $\mathrm{b}$ we show the spectral slope as a function of the phase angle in two ranges: between $0.55 \mu \mathrm{m}$ and $0.80 \mu \mathrm{m}$

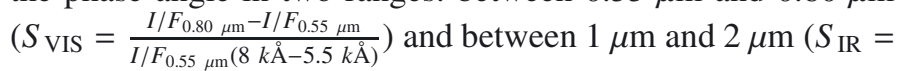

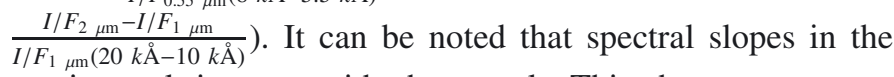
two intervals increase with phase angle. This phenomenon, typically referred to as phase reddening, is common to many planetary bodies, such as asteroids (Clark et al. 2002), icy satellites (Filacchione et al. 2012), and planetary rings (Filacchione et al. 2014), and has been also observed in laboratory measurements (Schröder et al. 2014). From the linear fits shown in Figs. 17a and $b$ we obtain values of relative phase reddening (angular coefficient divided by the intercept at $0^{\circ}$ ) of $0.44 \% / \mathrm{deg}$ in the VIS and $0.73 \% / \mathrm{deg}$ in the IR. A pronounced phase reddening is also reported in Fornasier et al. (2015) from the OSIRIS camera onboard Rosetta, where they measured a relative phase reddening in the VIS of about $0.95 \% / \mathrm{deg}$. This value is higher than ours, but it has been computed on a slightly different wavelength interval $(0.535-0.882 \mu \mathrm{m})$ and within a different solar phase angle range $1.3^{\circ}-53.9^{\circ}$. This latter argument can explain the difference between the two measurements, since the phase reddening intensity can vary with phase angle, as shown in Schröder et al. (2014).

In Fig. 17 we also show the same quantities after the photometric reduction. The dependence on phase angle has basically been removed, with final values of $S_{\text {VIS }}=0.20 / k \AA$ (Fig. 17c) and $S_{\text {IR }}=0.033 / k \AA$ (Fig. 17d). These quantities confirm the previous measurements of spectral slopes reported in Capaccioni et al. (2015). Phase reddening can be interpreted as an effect of multiple scattering (Cuzzi et al. 2002; Li et al. 2015), which is relatively stronger at large phase angles for bodies with a backscattering phase function, by stretching albedo differences at different wavelengths. This explanation is reasonable for surfaces with moderate to large $w$, where multiple scattering plays an important role, but may be not sufficient in the case of dark surfaces, where single scattering is the dominant process. In the photometric modeling presented so far we neglected multiple scattering; but our photometric reduction to $w$ removes phase reddening because the derived single scattering phase function behavior varies with wavelength (Fig. 11). It is then legitimate to wonder whether this result is just a compensation effect due to our simplified model. To test this hypothesis, we computed $I / F$ simulated slopes in the VIS and IR by applying a Hapke model including multiple scattering, with SSA values as derived by our analysis, but with constant values of the asymmetry factor in each spectral range $b_{\mathrm{VIS}}=-0.42$ and $b_{\mathrm{IR}}=-0.40$, compatible with the ones we determined so far, for all the observation geometries of our dataset (Fig. 18). This allows estimating to which extent phase reddening can be due to multiple scattering when the single scattering phase function is constant over the wavelength range. The values of phase reddening we obtain from a linear fit (red curve) of the simulated $I / F$ distribution of Fig. 18 are $0.1 \% / \mathrm{deg}$ for VIS and $0.14 \% / \mathrm{deg}$ for the IR, respectively, which is well below the ones we measure from VIRTIS observations. This indicates that for $67 \mathrm{P}$ and in more general terms for low-albedo surfaces, multiple scattering is not sufficient to explain phase reddening, and in particular, that single 

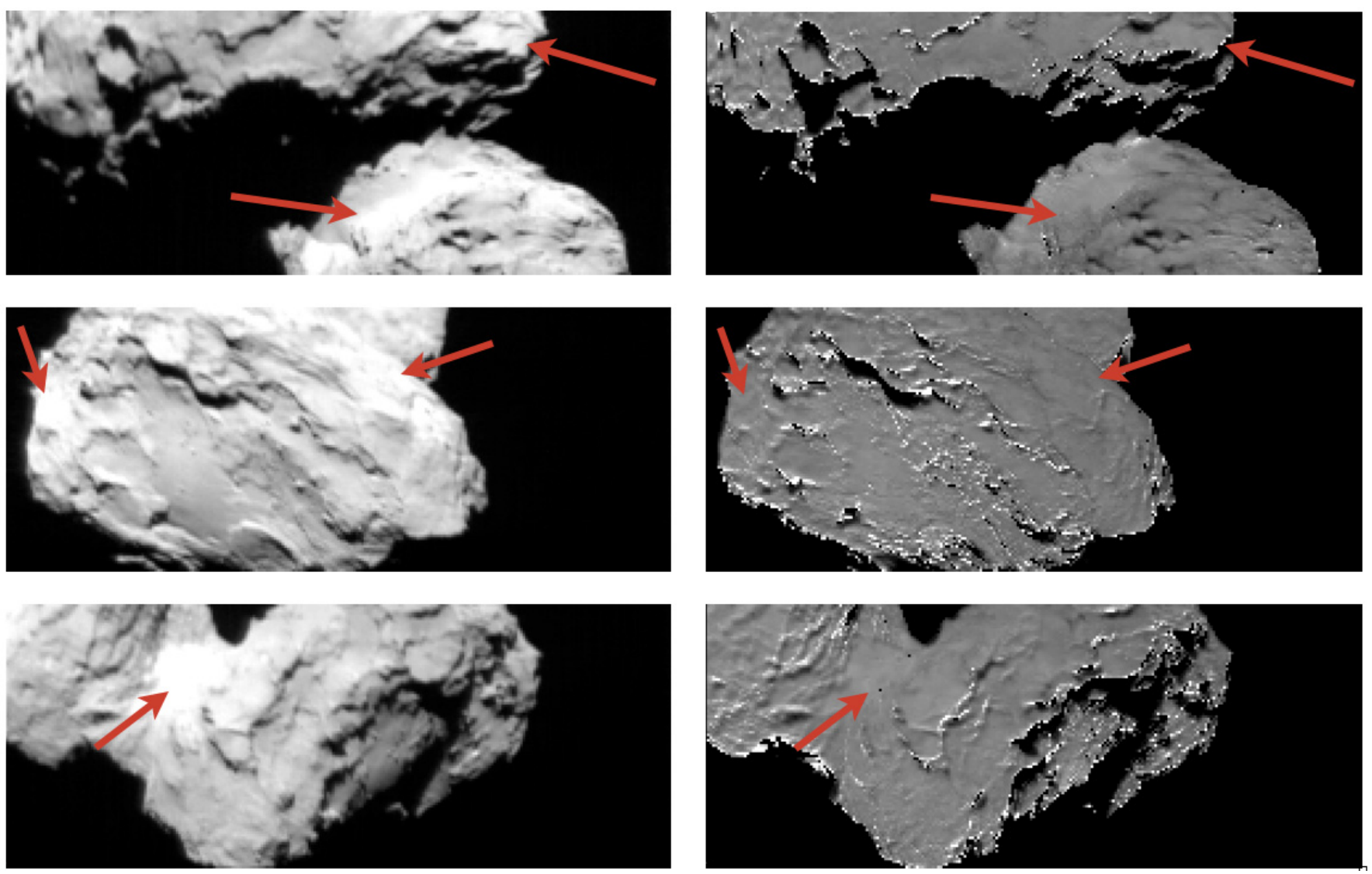

Fig. 16. $I / F$ images (left) and $w$ images after photometric reduction (right) at $0.55 \mu \mathrm{m}$. Red arrows indicate bright regions in the $I / F$ images. The same regions in the photometrically corrected images show a SSA level that is compatible with that of the surrounding terrains, revealing that $I / F$ brightness enhancement is due to observation geometry.

scattering is the dominant process. This also shows that the simplified Hapke model we adopted well describes the photometric properties of 67P.

Such a monotonic phase reddening has been measured and simulated by Schröder et al. (2014) for opaque material where single scattering is significant. They suggested that this effect might be related to the roughness on the surface of the particles composing the regolith combined to a positive spectral slope of the reflectance. This is compatible with our finding of a phase reddening related to the single scattering process. In fact, the SPPF, along with SSA, describes the average scattering property of the particle, and the behavior we derive (Fig. 11) can account for the effect of particle surface roughness.

\subsection{Albedo maps}

The derived SSA values (see Fig. 12) were reprojected, according to their coordinates, onto a cylindrical map with a grid of $1^{\circ}$ sampling both in longitude and latitude. If there was more than one value located in the same cell, we computed the median. The result of this approach are SSA maps of the surface, and in Fig. 19 we show two examples at $0.55 \mu \mathrm{m}$ and $1.8 \mu \mathrm{m}$. This allows investigating local photometric variability that cannot be appreciated from the global analysis presented so far because it is masked in the SSA albedo distribution derived in Fig. 15. To provide a reference, we divided the nucleus into four macro-regions (Fig. 19c): head, neck, body, and bottom. In the $0.55 \mu \mathrm{m}$ map (Fig. 19a) the neck region, which corresponds to the Hapi area of Thomas et al. (2015), and the center of the bottom region (Imothep in Thomas et al. 2015) have a relatively larger SSA than the rest of the comet, as found by Fornasier et al. (2015), while in the middle of the body region, approximately at $150^{\circ}<$ lon $<180^{\circ}, 40^{\circ}<$ lat $<60^{\circ}$, a darker area can be observed. Some residual of topography is still present, which produces the small-scale variations of the albedo that is due to the effects of partly shadowed pixels or miscorrection in the correspondence of sharp reliefs. A similar result is obtained for the map at $1.8 \mu \mathrm{m}$ (Fig. 19b). In this case, the Imothep plain appears even brighter than the neck, indicating a a slightly redder SSA across the VIS-IR. This is confirmed in Fig. 20, where a RGB composite map is shown from data at $0.44 \mu \mathrm{m}$ (blue), $0.55 \mu \mathrm{m}$ (green), and $0.70 \mu \mathrm{m}$ (red). A dichotomy of the nucleus surface can be noted, being redder in correspondence of the bottom, body, and head regions, while the neck area shows a more neutral color. This difference has not emerged from the full-disk photometric analysis (Sect. 2.3) because of the limited extension of the neck region as well as its position; it is frequently shadowed by the two main lobes of the nucleus.

\subsection{Zonal photometric reduction}

In this section we separately investigate the four macro-regions described in Sect. 3.5 by deriving an independent photometric reduction for each one. This approach has the twofold advantage of providing a more accurate photometric reduction for each region and exploiting, if present, global-scale differences in the spectrophotometric properties of the surface, which could have been masked in the previous analysis. In Fig. 21 we show the 


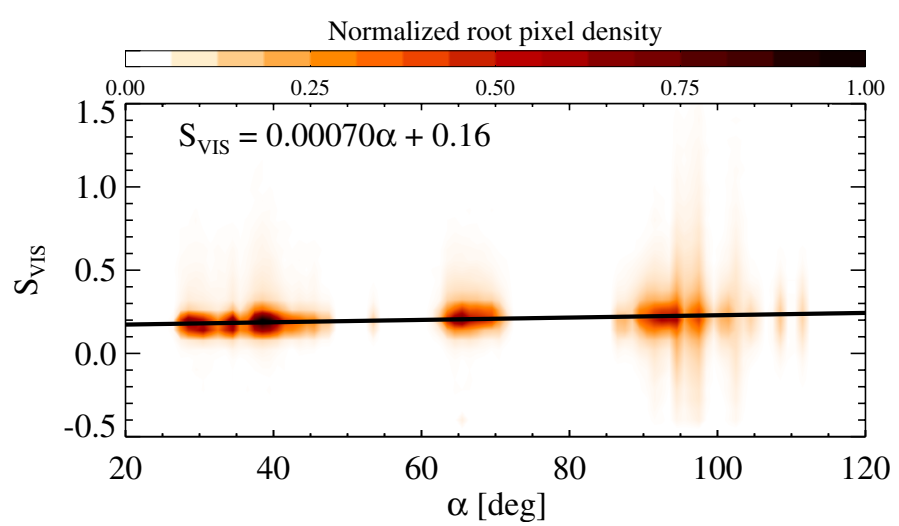

(a)

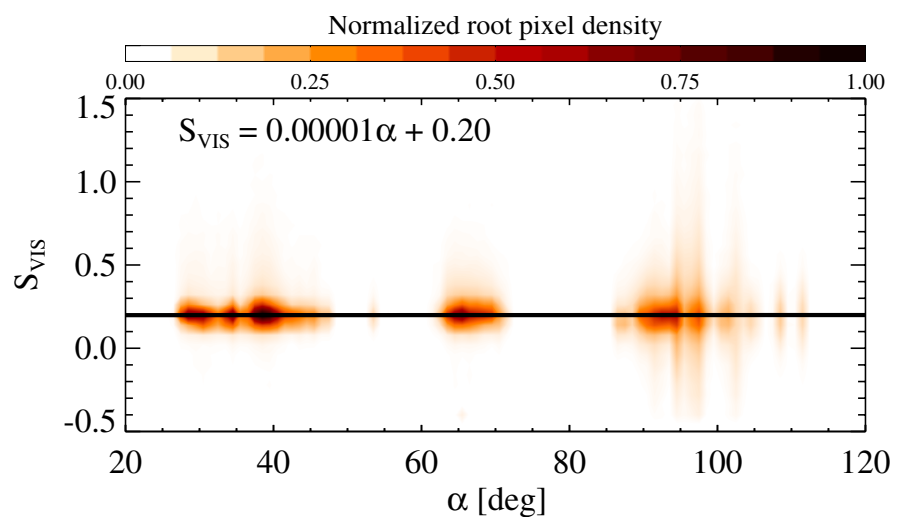

(c)

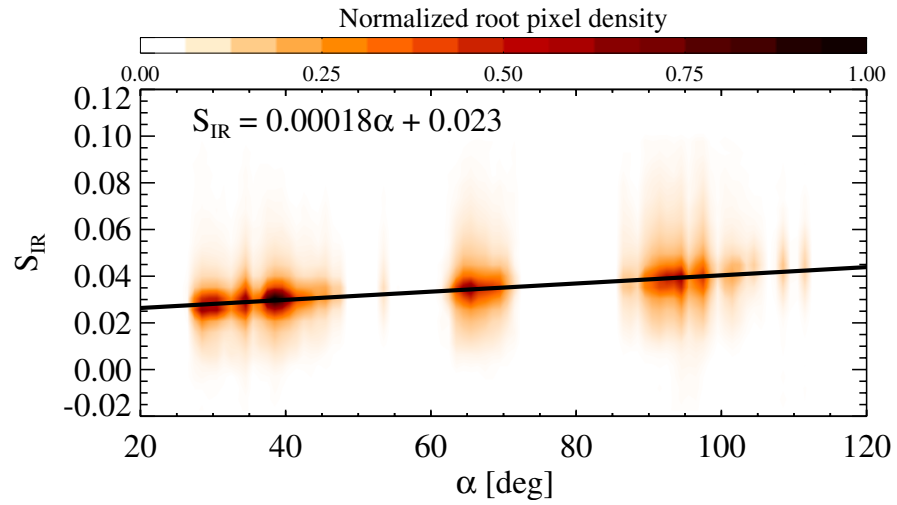

(b)

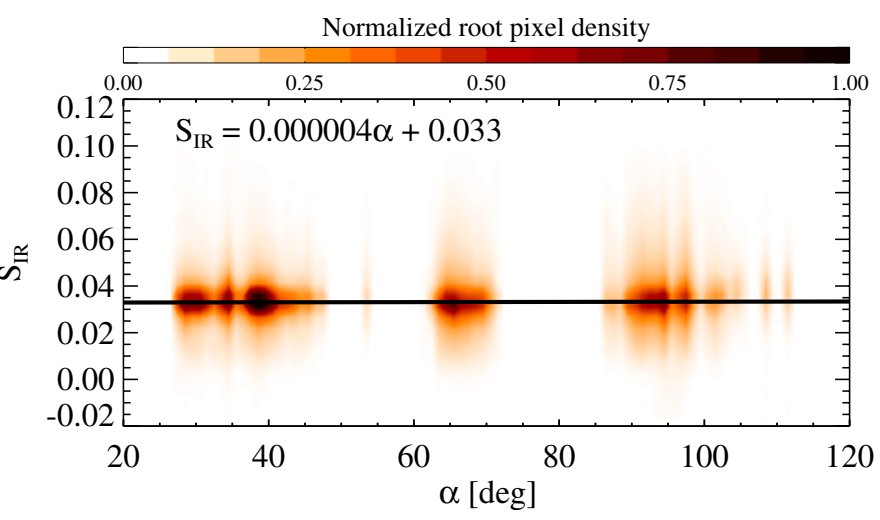

(d)

Fig. 17. Density plot of the spectral slope $\left[k \AA^{-1}\right]$ in the VIS range a) and IR b) range as a function of the phase angle. The same quantity, after photometric reduction, is shown in c) and d) for VIS and IR, respectively. Linear fits are drawn as a black line in the plots: the angular coefficient is expressed in $\left[k \AA^{-1} \mathrm{deg}^{-1}\right]$ and the intercept in $\left[k \AA^{-1}\right]$.

derived asymmetry parameter $b$ and the $w$ for the four macroregions. The derived spectra of $b$ and $w$ are similar to those derived for the global analysis of Sect. 3.1. The retrieved surface roughness parameters are $\bar{\theta}_{\text {head }}=21^{\circ}, \bar{\theta}_{\text {neck }}=18^{\circ}, \bar{\theta}_{\text {body }}=20^{\circ}$, and $\bar{\theta}_{\text {bottom }}=19^{\circ}$. The main differences with respect to the average properties of the nucleus are seen for the neck and the bottom region. In the first case, the derived parameters indicate a more backscattering SPPF and a deeper absorption feature at $3.2 \mu \mathrm{m}$, with the minimum shifted at $3.1 \mu \mathrm{m}$. The increased band depth at $3.2 \mu \mathrm{m}$ and the shift of the minimum can be due to an enrichment of water ice, which could also be related to the fact that the most of the activity is related to the region of the neck and surroundings at the time of these observations (Sierks et al. 2015). The bottom region, on the other side, shows the highest albedo in the IR. The derived roughness parameters are similar in the different regions and to the global value $\bar{\theta}=19^{\circ}$. This apparently contradicts the fact that the neck (Hapi) and the bottom (which includes the Imothep plain) are characterized by smooth terrains, as shown by OSIRIS images (Thomas et al. 2015), thus pointing to a link of the slope parameter with millimeter-scale roughness.

\section{Connecting full-disk and disk-resolved images}

In this section we present an attempt to retrieve a complete phase curve of the comet in the Bessel $V$-band (Bessell 1990) by connecting full-disk and disk-resolved data. For this purpose, we followed a classical approach in which the shape of the nucleus is assumed spherical: since the real shape is indeed not spherical, the accuracy of the resulting phase curve is limited by this assumption. The relative magnitude is determined from the integrated flux by means of Eq. (1). This is not possible in the case of disk-resolved images because a measurement of the integrated flux is not available. However, assuming that the nucleus is spherical, we can derive the flux from the comet in the $V$-band from the following equation:

$F_{67 P, V}=F_{\odot, V} \frac{\langle I / F\rangle_{\alpha, V}}{\pi} \frac{\pi R^{2} \Phi(\alpha)}{\Delta^{2}}$,

where $F_{\odot, V}$ is the solar flux, $\langle I / F\rangle_{\alpha, V}$ is the average reflectance at phase angle $\alpha$ from the dataset of disk-resolved images, $R$ is the comet equivalent radius, $\Phi(\alpha)=\frac{1+\cos (\alpha)}{2}$ is the fraction of the surface both visible and illuminated, and $\Delta$ is the spacecraftcomet distance. Given this, it is then possible to retrieve the reduced magnitude $V(1,1, \alpha)$ by applying the second and third expression of Eq. (1). In Fig. 22 we show the phase curve we obtained by combining the full-disk and disk-resolved datasets. The equivalent comet radius $R=1.72 \mathrm{~km}$ was estimated as the radius of a sphere having the same volume as comet 67P, which is $21.4 \pm 2.0 \mathrm{~km}^{3}$ (Sierks et al. 2015). At small phase angles the OE peak is fully developed, while at larger $\alpha$ the magnitude increases linearly. This ideally divides the curve into two regions with different linear phase coefficients, which were fitted with the following results: $\beta_{\alpha<15^{\circ}}=0.077 \pm 0.002 \mathrm{mag} /{ }^{\circ}$ and $\beta_{\alpha>15^{\circ}}=0.041 \pm 0.001 \mathrm{mag} /{ }^{\circ}$. We can compare $\beta_{\alpha<15^{\circ}}$ with previous measurements provided by Tubiana et al. (2011) with 
M. Ciarniello et al.: Photometric properties of comet 67P from VIRTIS-M aboard Rosetta

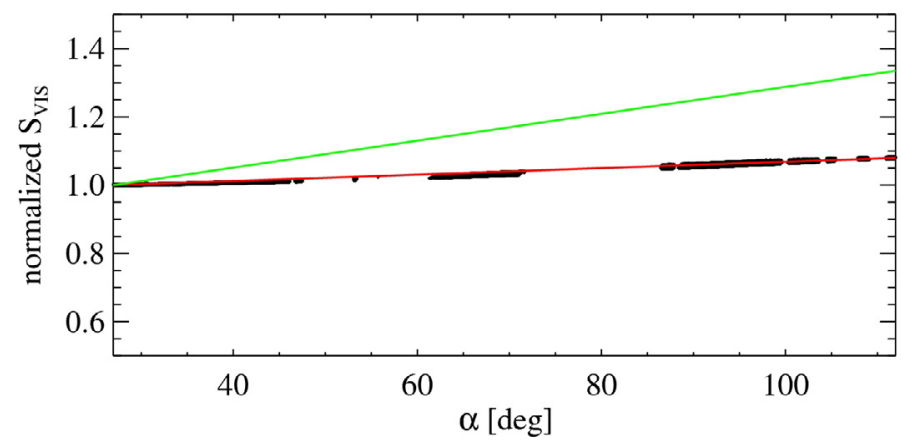

(a)

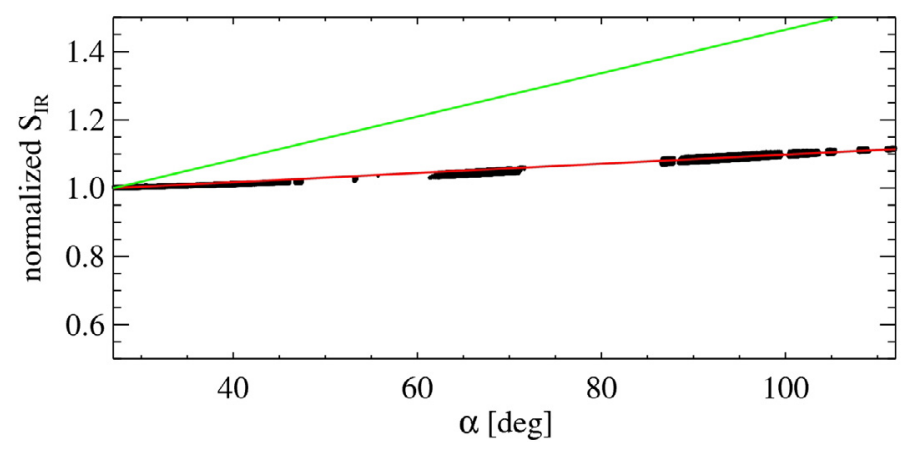

(b)

Fig. 18. $S_{\text {VIS }}$ a) and $S_{\text {IR }}$ b) normalized at the smallest phase angle as a function of $\alpha$. Black points are simulated values as described in the text, the red curve is their linear fit, and the green curve is the linear fit of the measured spectral slope distribution of comet 67P (Fig. 17).

compatible phase angle coverage. The values agree well and are for the latter in the range of $0.061-0.076 \mathrm{mag} /{ }^{\circ}$. With respect to other Jupiter-family comets, the value we retrieve is slightly higher than the average $\beta=0.053 \pm 0.005 \mathrm{mag} /{ }^{\circ}$ that has been derived by Snodgrass et al. (2011). Fornasier et al. (2015) computed a linear fit considering only points with $7^{\circ}<\alpha<54^{\circ}$ and derived $\beta=0.047 \pm 0.002 \mathrm{mag} /{ }^{\circ}$. This quantity is comparable to $\beta_{\alpha>15^{\circ}}$ derived in this work. The two quantities are slightly different, but it must be considered that the covered phase angle ranges are not the same in the two datasets and that our measurement assumes a spherical nucleus for this phase angle range. Fornasier et al. (2015) also derived the slope for $\alpha<7^{\circ}$ and obtained $\beta=0.074 \mathrm{mag} /{ }^{\circ}$, which perfectly agrees with $\beta_{\alpha<15^{\circ}}$ computed in this work. To further characterize the phase curve of 67P, we applied the HG formalism (Lagerkvist \& Magnusson 1990) to fit $V(1,1, \alpha)$. We obtained for the absolute magnitude $H_{V}(1,1, \alpha)=15.8 \pm 0.1$ and for the slope parameter $G=-0.09 \pm 0.04$, which both agree well with the values from OSIRIS measurements (Fornasier et al. 2015), $H_{V}(1,1, \alpha)=15.74 \pm 0.02$ and $G=-0.13 \pm 0.01$.

\section{Comparison to other comets}

Before the Rosetta mission, five comets have been imaged from instruments onboard spacecraft: 1P/Halley (Giotto), 9P/Tempel 1 (Deep Impact), 19P/Borrelly (Deep Space 1), 81P/Wild 2 (Stardust mission), and 103P/Hartley 2 (EPOXI). In this section we compare the photometric properties we derived for $67 \mathrm{P}$ with those derived for these comets and with previous measurements of 67P. In particular, here we compare color indices $B-V, V-R, R-I$, the SSA, the asymmetry parameter $b$, and the geometrical albedo $A_{\text {geo }}$ at $0.55 \mu \mathrm{m}$, the roughness parameter $\bar{\theta}$, and the linear phase coefficient $\beta$. The results are summarized in Table 3. Color index values of 67P were computed here after applying photometric reduction. This means that we assumed the flux of the comet to be proportional to the retrieved SSA. As shown in Sect. 3.4, the effect of phase reddening was eliminated, and this must be considered in the comparison with color indexes from other works, which are typically derived at a given observation geometry: generally low phase angles for ground-based observations and variable phase angles for measurements from spacecraft. The retrieved values of $B-V=0.73$ and $V-R=0.57$ are compatible with values of other comets. In particular, there is a good agreement with previous measurements of 67P by Tubiana et al. (2011) and Lamy et al. (2006). For the $R-I$ color index, our value of 0.59 is the highest of the comets reported in Table 3 , and in particular, it must be noted that it is not compatible with the one of Tubiana et al. (2011); this requires further investigation. The value of the 67P SSA is 0.052 at $0.55 \mu \mathrm{m}$ (from the distribution in Fig. 15a), which is higher than most of the values derived for other comets, but still indicates a very dark object, which appears to be a common property of cometary nuclei. The value derived in Fornasier et al. (2015) from disk-averaged analysis is $w=0.037$, but in their Hapke modeling OE is included and a lower value of the roughness parameter is assumed (Sect. 3.2). The asymmetry parameter $b=-0.42$ at $0.55 \mu \mathrm{m}$ indicates a backscattering SPPF, very similar to the other values reported in Table 3 and in perfect agreement with the value of Fornasier et al. (2015). The roughness slope parameter is comparable to other results, all indicating moderate to large topographical variegation, while the derived geometric albedo of $67 \mathrm{P}$ at $0.55 \mu \mathrm{m}$ is an intermediate value in this family of cometary nuclei, and our determination agrees with that of Sierks et al. (2015). Finally, we obtained two values of the linear phase coefficient, one for $\alpha<15^{\circ}$ and one for $\alpha>15^{\circ}$. As already mentioned, our determination at low phase angles is comparable with observation conditions of Tubiana et al. (2011) and the derivation of Fornasier et al. (2015) at $\alpha<7^{\circ}$, providing good agreement. Conversely, at large phase angles, our $\beta=0.041 \pm 0.001 \mathrm{mag} /{ }^{\circ}$ is the lowest of the entire set of values reported here. Fornasier et al. (2015) found an intermediate value in the phase angle range $7^{\circ}<\alpha<54^{\circ}$, close to our determination, but outside the error bars. We must recall in this context that the phase angle coverage plays an important role in determining the final value of $\beta$, and moreover, our determination relies on the strong assumption of a spherical shape of the comet. This makes a direct comparison with other measurements difficult.

\section{Conclusions and future work}

We have studied the photometric properties of the comet 67/P Churyumov-Gerasimenko as observed by the VIRTIS-M instrument onboard the Rosetta mission. The investigation has been developed in two parts, one concerning the analysis of fulldisk observations of the comet, and one based on disk-resolved images. For the full-disk observations we computed the integrated flux from the nucleus to derive the light curve of $67 \mathrm{P}$ at different phase angles. The light curve, as measured by VIRTIS, shows a double-peak behavior that is due to the elongated shape of the nucleus and agrees well with the one derived by Mottola et al. (2014). Since full-disk observations have been taken at low phase angles, we also preliminarily characterized the OE surge, suggesting shadow-hiding as the driving mechanism. 


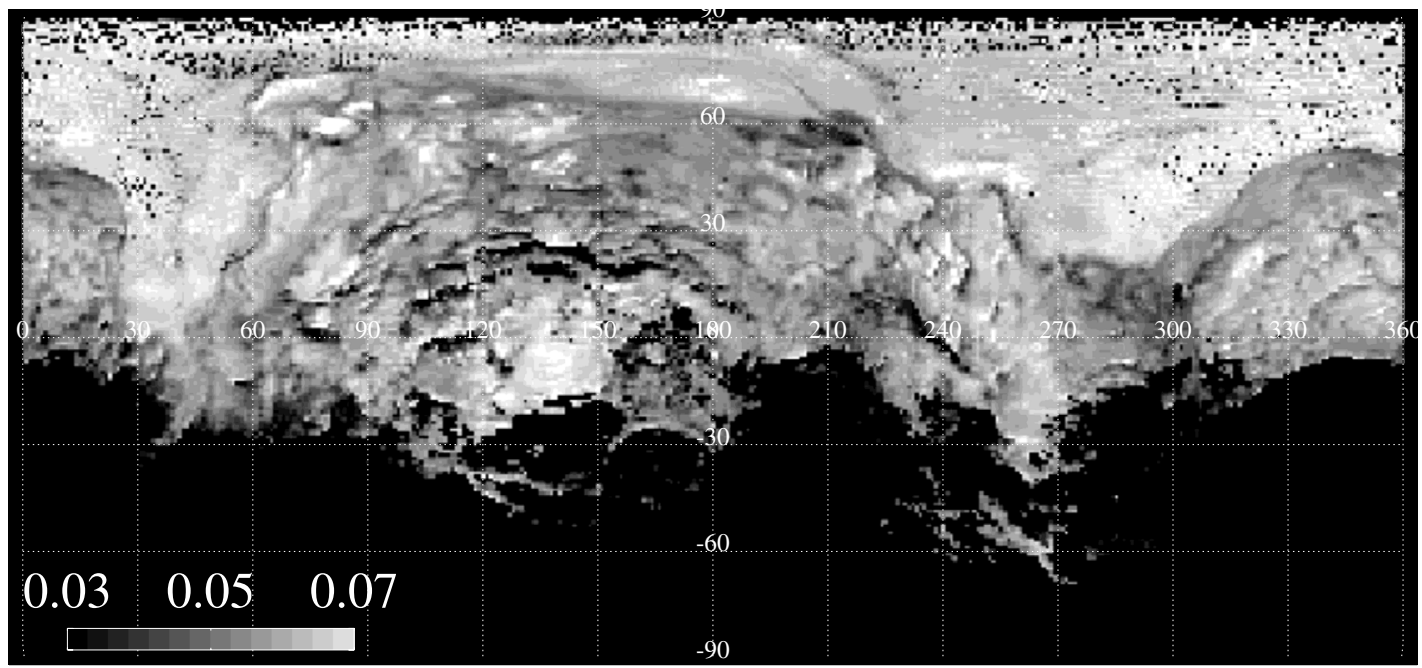

(a)

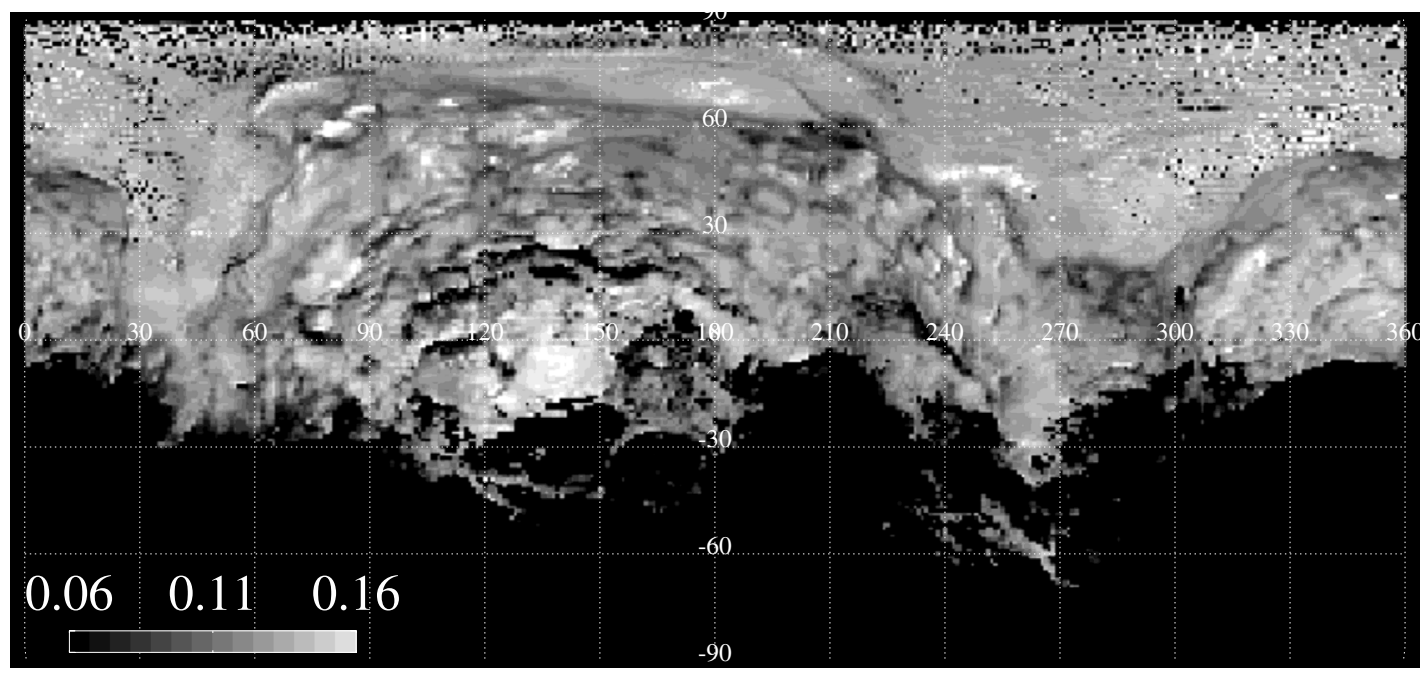

(b)

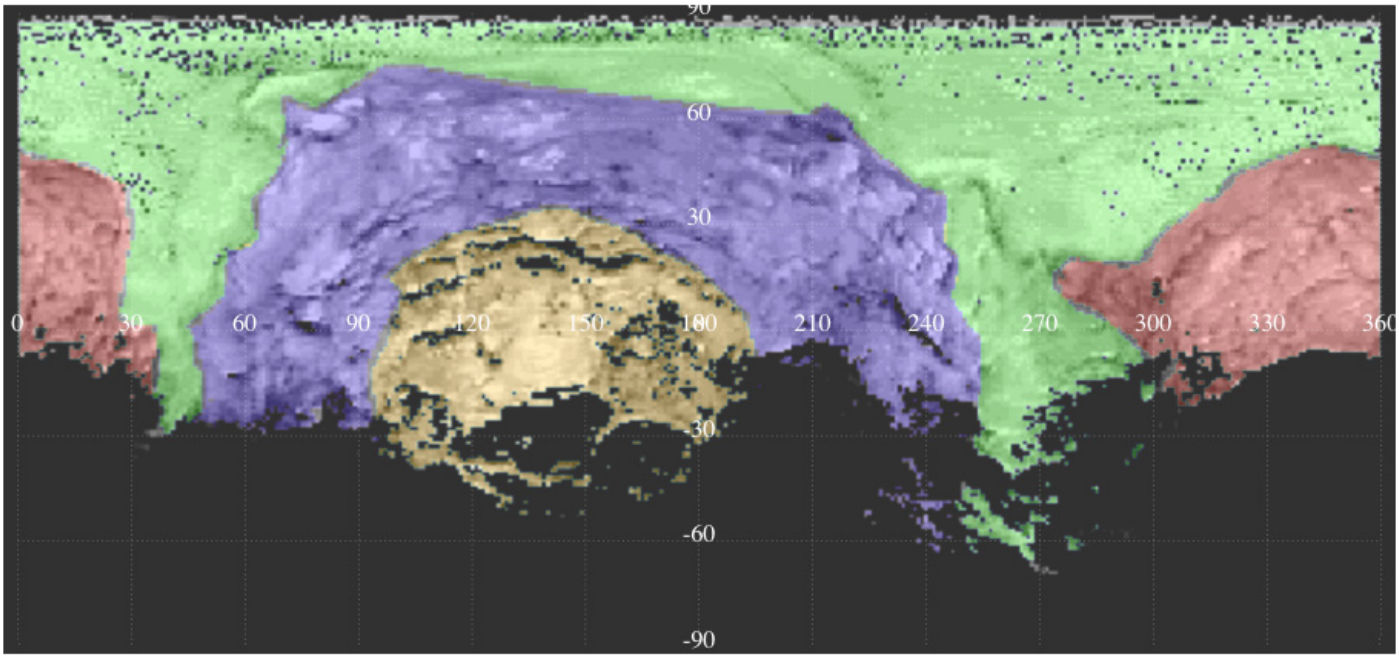

(c)

Fig. 19. a) SSA map at $0.55 \mu \mathrm{m}$. b) SSA map at $1.8 \mu \mathrm{m}$. c) The four macro-regions superimposed on the SSA albedo map: head (red), neck (green), body (blue), and bottom (orange). 


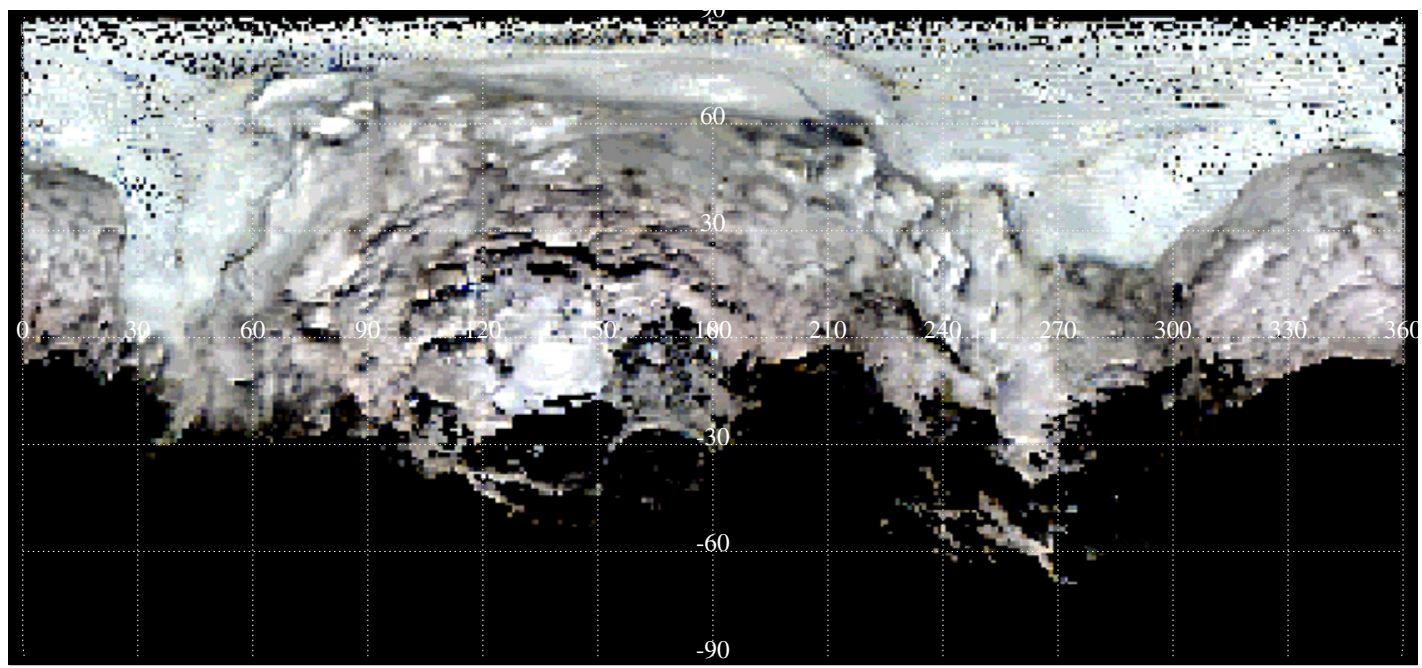

Fig. 20. Red $(0.70 \mu \mathrm{m})$, green $(0.55 \mu \mathrm{m})$, and blue $(0.44 \mu \mathrm{m})$ SSA map.

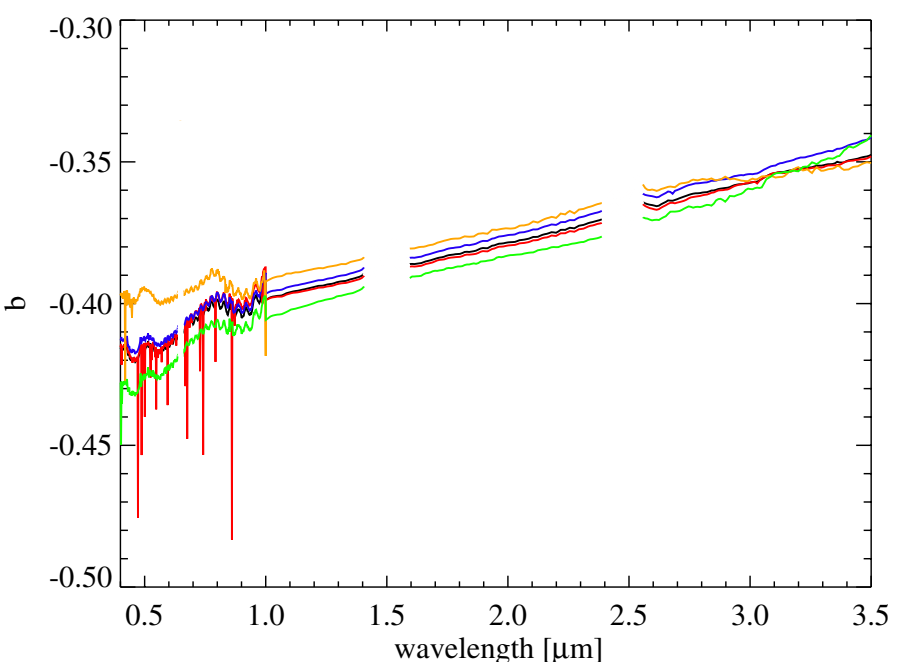

(a)

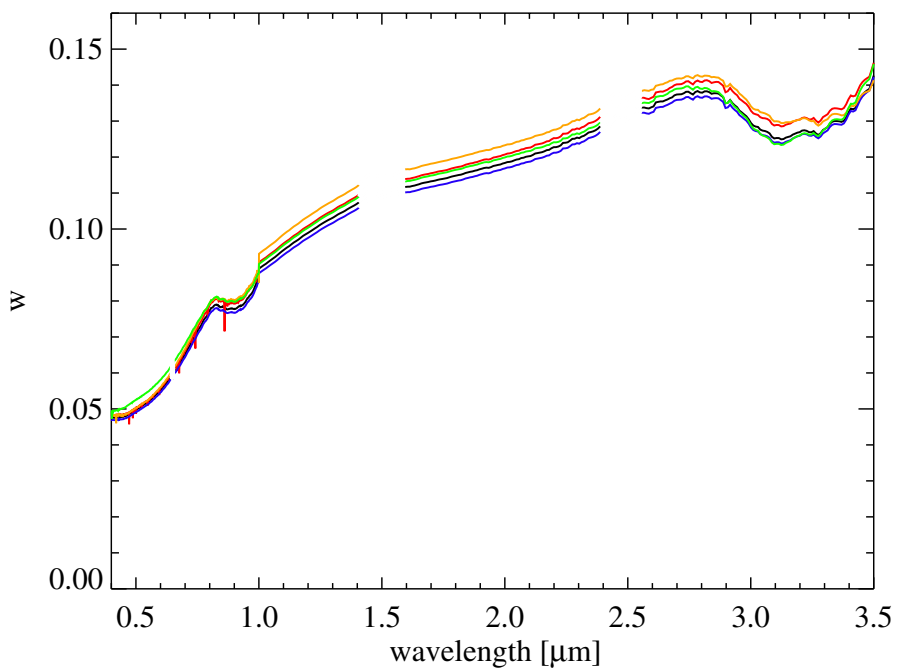

(b)

Fig. 21. Spectral variation of $b$ a) and $w \mathbf{b}$ ) for the four different macro-regions: head (red), neck (green), body (blue), and bottom (orange). The derived spectra from the global analysis are reported in black. The absorption common to all the spectra in the $0.8-1 \mu \mathrm{m}$ is likely a residual calibration artifact. Some abrupt oscillations of $w$ and $b$ at certain wavelengths are due to degeneration in the fitting.

The color ratios in the VIS and IR ranges were calculated to exploit large-scale compositional differences on the surface, with negative results. Taking advantage of full-disk data at very low phase $\left(\alpha<1^{\circ}-2.5^{\circ}\right)$, we estimated the normal albedo at $0.55 \mu \mathrm{m}$ and obtained $A_{\mathrm{n}}=0.062 \pm 0.002$. This value was assumed to match the geometric albedo $A_{\text {geo }}$, following the considerations of Sect. 2.4.

For the disk-resolved images, we used the huge dataset produced by VIRTIS-M so far to derive a photometric reduction to SSA and simultaneously determine the photometric properties of the nucleus. The photometric reduction we applied is a simplified Hapke model, where OE effect and multiple scattering were neglected because our phase angle coverage is limited to the range $27.2^{\circ}<\alpha<111.5^{\circ}$ and the surface of 67P is very dark. We retrieved a backscattering single particle phase function $(b=-0.42$ at $0.55 \mu \mathrm{m})$ and SSA $w=0.052 \pm 0.013$. The surface roughness was determined by the average slope parameter $19^{\circ}+4$.

We also investigated the phase-reddening effect. Our conclusion on this topic is that it is mainly due to single scattering through a wavelength-dependent phase function, and the role of multiple scattering can be neglected. We measured a phase reddening of $0.44 \% / \mathrm{deg}$ in the VIS, while for comparison, no reddening has been observed on Tempel 1 ( $\mathrm{Li}$ et al. 2007a). The color indices in the VIS range indicate that the spectral properties of 67P are typical in the context of cometary nuclei, except for $R-I$, which is larger than the average and indicates an increase of the reddening longward in the VIS.

Albedo maps were produced by means of photometrically reduced data. The neck (Hapi region) and the center of the bottom (Imothep plain) are sligthly brighter than the rest of the nucleus. The former shows a more neutral spectrum, while the latter appears redder, similarly to the rest of the surface (head and body).

A future development of this work will concern the inclusion of the $\mathrm{OE}$ in the modeling, taking advantage of the more recent disk-resolved acquisitions at low phase angles. We will also compare the photometric properties of the nucleus before and after the perihelion passage (13 August 2015) to exploit the modification of the photometric parameters induced by the cometary 
A\&A 583, A31 (2015)

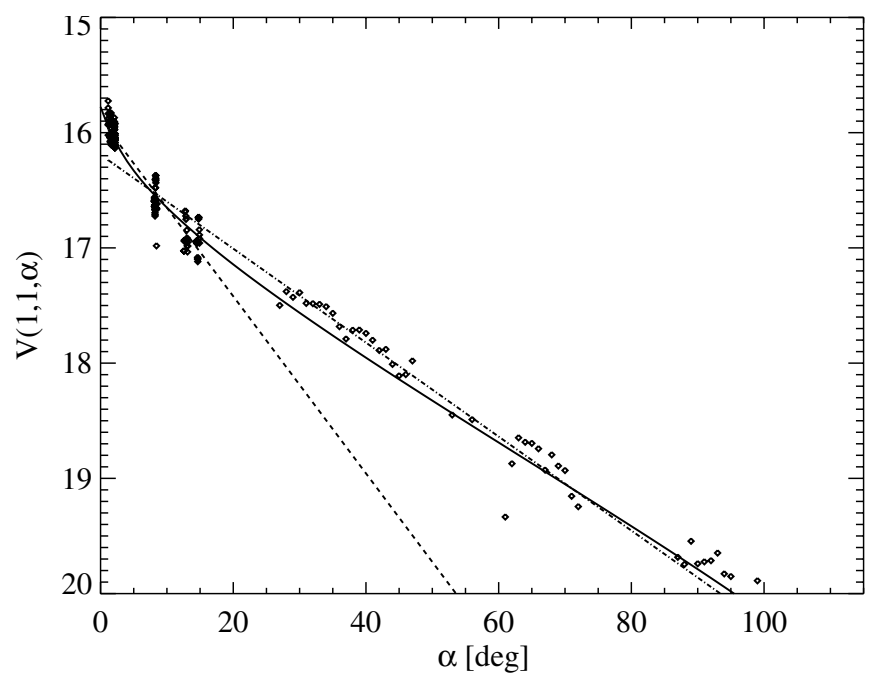

Fig. 22. $V$-band phase curve. Points with $\alpha<15^{\circ}$ are from full-disk images, while points with $\alpha>15^{\circ}$ are from disk-resolved data averaged on $1^{\circ}$ bins. Dashed and dot-dashed lines represent linear fits of $\alpha<15^{\circ}$ and $\alpha>15^{\circ}$ data points, respectively. The solid line is the fitted HG curve. Computed errors are typically smaller than the observed scatter of the points and are not shown in the plot because they are not representative of the real uncertainty. The scatter of points at $\alpha<15^{\circ}$, which correspond to integrated fluxes from VIRTIS data, is due to the variable projected cross section of the comet in the different observations. At larger solar phase angles the flux is computed from the average value of the $I / F$ at each given phase angle. The corresponding error, derived as the standard deviation of the $I / F$ distribution at each $\alpha$ normalized to the root of the number of elements in that phase angle bin, is much smaller than the observable scatter, indicating a non-Gaussian behavior.

activity to better constrain the composition and textural properties of the surface materials and their changes with time.

Acknowledgements. We thank the following institutions and agencies for support of this work: Italian Space Agency (ASI, Italy), Centre National d'Études Spatiales (CNES, France), DLR (Germany), NASA (USA) Rosetta Program, and Science and Technology Facilities Council (UK). VIRTIS was built by a consortium, which includes Italy, France, and Germany, under the scientific responsibility of the Istituto di Astrofisica e Planetologia Spaziali of INAF, Italy, which also guides the scientific operations. The VIRTIS instrument development, led by the prime contractor Selex-ES (Florence, Italy), has been funded and managed by ASI, with contributions from Observatoire de Meudon financed by CNES, and from DLR. We thank the Rosetta Science Ground Segment and the Rosetta Mission Operations Centre for their support throughout the early phases of the mission. The VIRTIS calibrated data will be available through the ESA's Planetary Science Archive Website (www.rssd.esa.int/index.php? project=PSA\&page=index) and is available upon request until posted to the archive. The VIRTIS Team wishes to dedicate this paper to the memory of Angioletta Coradini, conceiver of the instrument, our leader, and friend.

\section{References}

Akimov, L. A. 1988, Kinematika i Fizika Nebesnykh Tel, 4, 3

Bessell, M. S. 1986, ASP Pub., 98, 1303

Bessell, M. S. 1990, ASP Pub., 102, 1181

Buratti, B. J., Hicks, M. D., Soderblom, L. A., et al. 2004, Icarus, 167, 16

Capaccioni, F., Coradini, A., Filacchione, G., et al. 2015, Science, 347, 628

Ciarniello, M., Capaccioni, F., Filacchione, G., et al. 2011, Icarus, 214, 541

Ciarniello, M., Capaccioni, F., \& Filacchione, G. 2014, Icarus, 237, 293

Clark, B. E., Helfenstein, P., Bell, J. F., et al. 2002, Icarus, 155, 189

Coradini, A., Capaccioni, F., Drossart, P., et al. 2007, Space Sci. Rev., 128, 529

Cuzzi, J. N., French, R. G., \& Dones, L. 2002, Icarus, 158, 199

Domingue, D. L., Lockwood, G., \& Thompson, D. 1995, Icarus, 115, 228

Filacchione, G., Capaccioni, F., Ciarniello, M., et al. 2012, Icarus, 220, 1064

Filacchione, G., Ciarniello, M., Capaccioni, F., et al. 2014, Icarus, 241, 45

Fornasier, S., Hasselmann, P. H., Barucci, M. A., et al. 2015, A\&A, 583, A30

Hapke, B. 1993, Theory of reflectance and emittance spectroscopy, Topics in Remote Sensing (Cambridge, UK: Cambridge University Press), 3

Hapke, B. 2002, Icarus, 157, 523

Hapke, B. 2008, Icarus, 195, 918

Hapke, B. 2012, Theory of reflectance and emittance spectroscopy (Cambridge University Press)

Hapke, B., Nelson, R., \& Smythe, W. 1998, Icarus, 133, 89

Heyney, L. G., \& Greenstein, J. L. 1941, ApJ, 93, 70

Kaydash, V., Pieters, C., Shkuratov, Y., \& Korokhin, V. 2013, J. Geophys. Res. (Planets), 118, 1221

Keller, H. U., Barbieri, C., Lamy, P., et al. 2007, Space Sci. Rev., 128, 433

Lagerkvist, C.-I., \& Magnusson, P. 1990, A\&A, 86, 119

Lamy, P. L., Toth, I., Weaver, H. A., et al. 2006, A\&A, 458, 669

Li, J.-Y., A'Hearn, M. F., Farnham, T. L., \& McFadden, L. A. 2009, Icarus, 204, 209

Li, J.-Y., A'Hearn, M. F., Belton, M. J. S., et al. 2007a, Icarus, 187, 41

Li, J.-Y., A'Hearn, M. F., McFadden, L. A., \& Belton, M. J. S. 2007b, Icarus, 188,195

Li, J.-Y., Besse, S., A'Hearn, M. F., et al. 2013, Icarus, 222, 559

Li, J.-Y., Helfenstein, P., Buratti, B. J., Takir, D., \& Clark, B. E. 2015, Asteroid IV Book, submitted [arXiv: 1502 .06302]

Longobardo, A., Palomba, E., Capaccioni, F., et al. 2014, Icarus, 240, 20

Lowry, S. C., Fitzsimmons, A., \& Collander-Brown, S. 2003, A\&A, 397, 329

Minnaert, M. 1941, ApJ, 93, 403

Mottola, S., Lowry, S., Snodgrass, C., et al. 2014, A\&A, 569, L2

Preusker, F., Scholten, F., \& Matz, K.-D. 2015, A\&A, 583, A33

Raponi, A. 2015, Ph.D. Thesis [arXiv: 1503.08172]

Schröder, S. E., Grynko, Y., Pommerol, A., et al. 2014, Icarus, 239, 201

Shepard, M. K., \& Helfenstein, P. 2011, Icarus, 215, 526

Shkuratov, Y., Kreslavsky, M. A., Ovcharenko, A. A., et al. 1999, Icarus, 141, 132

Sierks, H., Barbieri, C., Lamy, P. L., et al. 2015, Science, 347, 1044

Snodgrass, C., Fitzsimmons, A., Lowry, S. C., \& Weissman, P. 2011, MNRAS, 414,458

Thomas, N., \& Keller, H. U. 1989, A\&A, 213, 487

Thomas, N., Sierks, H., Barbieri, C., et al. 2015, Science, 347, 440

Tubiana, C., Böhnhardt, H., Agarwal, J., et al. 2011, A\&A, 527, A113 